\title{
ON THE STABILITY OF MONOPOLE SOLUTIONS
}

\author{
GEORGE ANDROULAKIS AND STAMATIS DOSTOGLOU
}

\begin{abstract}
We study the Hessian of the Yang-Mills-Higgs functional as the self-interaction parameter $\lambda$ varies and examine how convergence in the configuration space controls the sign of the first eigenvalue at the limit. As an application, we show that the spherically symmetric solutions of 't Hooft and Polyakov are stable for $\lambda$ in the neighborhood of 0 and that the kernels of their Hessians are precisely the spaces generated by their spatial derivatives.
\end{abstract}

\section{INTRODUCTION}

In 1966 Higgs [H] introduced a Lagrangian coupling vector gauge fields with a pair of scalar fields and inducing spontaneous symmetry breaking. In this way, the fields of the model acquired masses, which corresponded to exponential decay. The Yang-Mills-Higgs functional $E_{\lambda}$ on $\mathbf{R}^{3}$ is the static, classical version of Higgs' functional and describes massive particles with magnetic charge. These are the magnetic monopoles. The massive components of the Higgs field $\Phi$ have mass bounded by $\sqrt{\lambda}$, for $\lambda$ a parameter in the functional.

The Prasad-Sommerfield limit of the theory is obtained by setting $\lambda=0$. Inevitably, at this limit some exponential decay is lost. The idea is that information at the limit ought to propagate to the $\lambda \neq 0$ case, and trying to substantiate this has been the main motivation for this paper. A unique feature of the $\lambda=0$ limit is that the minima satisfy first order equations, the Bogomo'lnyi equations that, of course, imply the second order variational equations for $\lambda=0$. In fact, Prasad and Sommerfield gave closed form solutions for these minima when the magnetic charge is 1 . For $\lambda=0$ it is now known that there exist minimal solutions for any magnetic charge $[\mathrm{JT}]$, as well as non-minimal solutions of arbitrarily high energy [T3]. 
On the other hand, spherically symmetric solutions of the variational equations of $E_{\lambda}$, for any $\lambda \geq 0$, were suggested by 't Hooft and Polyakov $[\mathrm{tH}],[\mathrm{P}]$. For each $\lambda$ these are obtained by minimizing $E_{\lambda}$ amongst all spherically symmetric configurations. For $\lambda=0$ the 't Hooft-Polyakov solutions are precisely the Prasad-Sommerfiled solutions of the Bogomol'nyi equations and hence minima.

It has however not been known whether for $\lambda>0$ the 't Hooft-Polyakov solutions are minima, either global or local. In fact, it is still not known whether each component of the configuration space does have minima of $E_{\lambda}$, just as it does for $E_{0}$. Even more mysteriously, a minimum over all components exists in an as yet unknown component [ST]. The main application of this paper is:

Theorem 5.6 For positive $\lambda$ in a neighborhood of 0 the 't Hooft Polyakov spherically symmetric solution is stable. The kernel of the corresponding Hessian consists entirely of translation modes.

Stability here is the same as in [BL]: the index of the Hessian $Q_{\lambda}$ at the solution $c_{\lambda}$ is zero. However, the methods here provide control also on the Kernel of $Q_{\lambda}$. Therefore the absence of energy decreasing directions is also established.

In general, minimizers amongst suitably symmetric configurations will always be critical points. However they will not necessarily be minimizers (either local or global) over all configurations. For an energy functional where the spherically symmetric minimizer is overall unstable see the Skyrmion functional in [WB].

The proof of the main result has two parts:

1. It is first shown that $c_{\lambda}$ converges to $c_{0}$ in the configuration space and therefore for small $\lambda$ the difference between the Hessians $Q_{\lambda}$ and $Q_{0}$ is small. This convergence follows from energy bounds and control of the first derivatives of the fields.

2. It is then shown that if $v$ is orthogonal to the translation modes of the $\lambda$-solution for some small $\lambda$, then $v$ will stay almost orthogonal to the kernel of $Q_{0}$. For this one needs control on the second derivatives of the fields. Although for different $\lambda$ 's the $Q_{\lambda}$ 's are defined on different Hilbert spaces, the convergence of step (1) allows isomorphisms 
between them and therefore orthogonality will always be with respect to the same inner product. This step is formalized by defining "subspaces containing the kernel of $Q_{0}$ at the limit".

With these established, if $v$ is a decreasing direction for $E_{\lambda}$ it would also have to be decreasing for $E_{0}$, by (1). Since the $\lambda=0$ solution is a minimum, $Q_{0}$ has no negative eigenvalues. What could still happen is that $v$ could end up in the kernel of $Q_{0}$. This cannot happen by (2).

Section 2 describes the set-up. Section 3 presents the main results for quadratic forms in general. Section 4 applies section 3 to the Yang-Mills-Higgs Hessians. Section 5 applies section 4 to spherically symmetric solutions.

All estimates needed for steps (1) and (2) above for the spherically symmetric solutions are first outlined in 6 and then provided in sections 7 to 10. The estimates are then put together in Section 11. Section 12 presents some arguments for all $\lambda$, not necessarily close to 0 .

The estimates obtained for spherically symmetric solutions are uniform in $\lambda$ and sharper than the estimates known to hold for arbitrary critical points of the Yang-Mills-Higgs functional, $[\mathrm{JT}]$. Some effort has been made to keep control of the range of $\lambda$ for which the statements are valid. The first restriction appears in Proposition 9.1, where $\lambda$ has to be chosen so that a certain coefficient is negative. The second restriction happens in Step 2 of Proposition 10.1, where bounds needed for certain norms to be uniformly bounded do not come as a direct consequence of bounded energies.

As usual, $C$ will denote a generic constant, with value possibly changing from line to line.

\section{SET-UP}

The classical Yang-Mills-Higgs action functional $E_{\lambda}$ with self-interaction parameter $\lambda \geq 0$ is defined by

$$
E_{\lambda}(A, \Phi)=\frac{1}{2} \int_{\mathbf{R}^{3}}\left\{\left|F_{A}\right|^{2}+\left|d_{A} \Phi\right|^{2}+\frac{\lambda}{4}\left(|\Phi|^{2}-1\right)^{2}\right\} d^{3} x,
$$

on pairs $c=(A, \Phi)$. Here $A$ is a connection on the $S U(2)$ bundle $S U(2) \times \mathbf{R}^{3}$ over $\mathbf{R}^{3}$. $\Phi$ is a section of the associated bundle $E$ with fibre the Lie Algebra $\mathfrak{s u}(2), E=\mathfrak{s u}(2) \times \mathbf{R}^{3}$. $F_{A}$ 
is the curvature of the connection $A$ :

$$
F_{A}=d A+\frac{1}{2}[A, A]
$$

and $d_{A}$ the covariant derivative of $\Phi$ with respect to the connection $A$

$$
d_{A} \Phi=d A+[A, \Phi]
$$

All inner products are with respect to the Killing inner product on $\mathfrak{s u}(2)$ and the standard metric on $\mathbf{R}^{3}$.

The Prasad-Sommerfeld limit is the corresponding functional for $\lambda=0$ :

$$
E_{0}(A, \Phi)=\frac{1}{2} \int_{\mathbf{R}^{3}}\left\{\left|F_{A}\right|^{2}+\left|d_{A} \Phi\right|^{2}\right\} d^{3} x,
$$

defined on the configuration space

$$
\hat{\mathcal{C}}=\left\{(A, \Phi): A \in L_{1, l o c}^{2}, \Phi \in L_{1, l o c}^{2}, E_{0}(A, \Phi)<\infty\right\}
$$

equipped with the $L_{1, l o c}^{2}$ topology intersected with the topology that makes $\left\|d_{A} \Phi\right\|_{2}$ and $\left\|F_{A}\right\|_{2}$ continuous. With respect to this topology, $\hat{\mathcal{C}}$ consists of countably many connected components labeled by the integer monopole number

$$
N=\frac{1}{4 \pi} \int_{\mathbf{R}^{3}} \operatorname{Tr}\left(F_{A} \wedge d_{A} \Phi\right)
$$

Now for any $\lambda$, all configurations $(A, \Phi)$ with $E_{\lambda}(A, \Phi)<\infty$ clearly lie in $\hat{\mathcal{C}}$. However, the third term in $E_{\lambda}(A, \Phi)$ is not always finite for $c$ in $\hat{\mathcal{C}}$. For this, define

$$
\hat{\mathcal{C}}_{+}=\left\{c \in \mathcal{C}: E_{\lambda}(c)<\infty, \text { for some } \lambda>0\right\}
$$

and consider on it the topology it acquires as a subset of $\hat{\mathcal{C}}$.

In addition, $E_{\lambda}, \lambda \geq 0$ is invariant under the action of the gauge group

$$
\mathcal{G}=\left\{g: \mathbf{R}^{3} \rightarrow \mathrm{SU}(2), \quad \mathrm{g} \in \mathrm{L}_{2, \mathrm{loc}}^{2}\right\}
$$

where

$$
g \cdot A=g A g^{-1}+g d g^{-1}, \quad g \cdot \Phi=g \Phi g^{-1} .
$$

In reality then $E_{0}$ and $E_{\lambda}$ are defined on the quotients

$$
\mathcal{C}=\hat{\mathcal{C}} / \mathcal{G}, \quad \mathcal{C}_{+}=\hat{\mathcal{C}}_{+} / \mathcal{G}
$$


respectively. It follows from Part 1 of Theorem 5.5 below that $\mathcal{C}_{+}$is not closed in $\mathcal{C}$.

To define the space of infinitesimal perturbations at $c=(A, \Phi)$ in $\mathcal{C}$, start by defining $H_{c}$ to be the completion of $C_{0}^{\infty}$ sections with respect to the inner product norm

$$
\|(a, \phi)\|_{c}^{2}=\left\|\nabla_{A} a\right\|_{2}^{2}+\left\|\nabla_{A} \phi\right\|_{2}^{2}+\|[\Phi, a]\|_{2}^{2}+\|[\Phi, \phi]\|_{2}^{2}
$$

which allows for finite $E_{0}$ directions only, c.f. [T2]. In addition the norm also fixes the behavior of $\Phi$ at infinity by excluding the directions

$$
(a(x), \phi(x))=\left.\frac{d}{d \sigma}\right|_{\sigma=1}(\sigma A(\sigma x), \sigma \Phi(\sigma x)) .
$$

With respect to the $L_{2}$-inner product the $(a, \phi)$ 's that are perpendicular to the gauge orbit of $c=(A, \Phi)$ must satisfy

$$
\partial_{c}(a, \phi):=d_{A}^{*} a+[\Phi, \phi]=0 .
$$

However, since all $L_{2, l o c}^{2}$ gauge transformations are allowed, for $\lambda=0$ the Kernel of $\partial_{c}$ still contains a gauge direction, namely $\left(d_{A} \Phi, 0\right)$. Hence for $\lambda=0$ the space of admissible perturbations at $c$ will be isometric to

$$
T_{c} \mathcal{C}=\left\{(a, \phi) \in H_{c}: \partial_{c}(a, \phi)=0,(a, \phi) \perp_{c}\left(d_{A} \Phi, 0\right)\right\}
$$

where $\perp_{c}$ indicates perpendicularity with respect to $<,>_{c}$. It is a fact that with these conventions $\mathcal{C}$ can be thought of as a Banach manifold with tangent space $T_{c} \mathcal{C}$, see $[\mathrm{F}]$.

If $E_{\lambda}(A, \Phi)<\infty$ it will be useful to distinguish those directions in $T_{c} \mathcal{C}$ that keep $E_{\lambda}$ finite. For this, define the subspace of $T_{c} \mathcal{C}$

$$
T_{c} \mathcal{C}_{+}=\left\{(a, \phi) \in T_{c} \mathcal{C}:\|\phi\|_{2}<\infty .\right\}
$$

It is also a fact that in this way $\mathcal{C}_{+}$also a Banach manifold with tangent space $T_{c} \mathcal{C}_{+}$, see [D1].

The variational equations for $\lambda \geq 0$ are the Yang-Mills-Higgs equations

$$
\begin{gathered}
d_{A}^{*} F_{A}=\left[d_{A} \Phi, \Phi\right], \\
d_{A}^{*} d_{A} \Phi=-\frac{\lambda}{2} \Phi\left(|\Phi|^{2}-1\right) .
\end{gathered}
$$


Note that for $\lambda=0$ the functional can be written as

$$
E_{0}(A, \Phi)=\left\|F_{A} \mp * d_{A} \Phi\right\|_{2}^{2} \pm 4 \pi N
$$

for $N$ defined by (1), which shows why for the unique case $\lambda=0$ the minima in each connected component satisfy the first order Bogomo'lnyi equation

$$
\pm F_{A}=* d_{A} \Phi
$$

For any $c=(A, \Phi)$ in $\mathcal{C}$, the second derivative of the energy $E_{\lambda}$ defines a bilinear form on $H_{c}$

$$
\hat{Q}_{\lambda}^{c}\left(\left(a_{1}, \phi_{1}\right),\left(a_{2}, \phi_{2}\right)\right)=\frac{d^{2}}{d s d t} E_{\lambda}\left(A+s a_{1}+t a_{2}, \Phi+s \phi_{1}+t \phi_{2}\right), \quad \lambda \geq 0 .
$$

Calculate that

$$
\begin{aligned}
\hat{Q}_{\lambda}^{c}\left(\left(a_{1}, \phi_{1}\right),\left(a_{2}, \phi_{2}\right)\right) & =<F_{A},\left[a_{1}, a_{2}\right]>+<d_{A} \Phi,\left[a_{1}, \phi_{2}\right]+\left[a_{2}, \phi_{1}\right]>+<d_{A} a_{1}, d_{A} a_{2}> \\
& +<d_{A} \phi_{1}, d_{A} \phi_{2}>+<\left[a_{1}, \Phi\right],\left[a_{2}, \Phi\right]>+<d_{A} \phi_{1},\left[a_{2}, \Phi\right]> \\
& +<d_{A} \phi_{2},\left[a_{1}, \Phi\right]>+\frac{\lambda}{2} \int_{\mathbf{R}^{3}}\left(|\Phi|^{2}-1\right)<\phi_{1}, \phi_{2}>d^{3} x \\
& +\lambda \int_{\mathbf{R}^{3}}<\Phi, \phi_{1}><\Phi, \phi_{2}>d^{3} x .
\end{aligned}
$$

and the corresponding quadratic form

$$
\begin{aligned}
Q_{\lambda}^{c}(a, \phi) & =<F_{A},[a, a]>+2<d_{A} \Phi,[a, \phi]>+\left\|d_{A} a\right\|_{2}^{2} \\
& +\left\|d_{A} \phi\right\|_{2}^{2}+\|[a, \Phi]\|_{2}^{2}+2<d_{A} \phi,[a, \Phi]> \\
& +\frac{\lambda}{2} \int_{\mathbf{R}^{3}}\left(|\Phi|^{2}-1\right)|\phi|^{2} d^{3} x+\lambda \int_{\mathbf{R}^{3}}<\Phi, \phi>^{2} d^{3} x .
\end{aligned}
$$

The importance of $Q_{\lambda}^{c}$ becomes clear when one writes the Taylor expansion of $E_{\lambda}$ :

$$
E_{\lambda}(c+t v)=E_{\lambda}(c)+t\left(\nabla E_{\lambda}\right)_{c}(v)+\frac{t^{2}}{2} Q_{\lambda}^{c}(v)+o\left(t^{2}\right)
$$

Then if $c$ is a critical point, $v$ 's on which $Q_{\lambda}^{c}(v)$ is negative correspond to energy decreasing directions. According to Lemma 6.6 of [T1] this corresponds to eigenvectors with negative eigenvalue with respect to the norm above.

The aim now will be to investigate the behavior of $Q_{\lambda}^{c_{i}}$ as $c_{i}$ tends to $c_{0}$ in $\mathcal{C}$, especially when the $c_{i}$ 's are in $\mathcal{C}_{+}$but the limit $c_{0}$ lies in $\mathcal{C} \backslash \mathcal{C}_{+}$. For this it is useful to digress from 
the Yang-Mills-Higgs Theory for a while and investigate some properties of quadratic forms in general.

\section{Quadratic Forms}

Let $\hat{Q}: H \times H \rightarrow \mathbf{R}$ be a symmetric bilinear form on a Hilbert space $(H,<,>)$ with associated quadratic form $Q: H \rightarrow \mathbf{R}$,

$$
Q(v)=\hat{Q}(v, v)
$$

for $v$ in $H$. If $Q$ is a quadratic form, then $\hat{Q}$ will denote the unique (by the polarization identity) symmetric bilinear form that satisfies (4).

For a quadratic form $Q$, define the linear subspace of $H$

$$
\operatorname{Ker} Q:=\{v \in H: \hat{Q}(v, w)=0 \text { for all } w \in H\}
$$

Now let $v$ be such that $Q(v)<0$ and let $P$ be the projection on the $\operatorname{Ker} Q$. Then writing $v$ as $v=P v \oplus(I-P) v$ and noting that by the definition of $\operatorname{Ker} Q$ and the symmetry of $\hat{Q}$

$$
\hat{Q}(v, v)=\hat{Q}((I-P) v,(I-P) v)
$$

one needs only consider those $v$ 's that make $Q$ negative and are perpendicular to $\operatorname{Ker} Q$.

Recall that a quadratic form is weakly lower semi-continuous if $v_{n} \rightarrow v_{0}$ weakly in $H$ implies $Q\left(v_{0}\right) \leq \lim \inf Q\left(v_{n}\right)$. For example, on any Hilbert space $v \mapsto\|v\|^{2}$ is weakly lower semi-continuous. To examine the properties of quadratic forms that will be important for the Hessians of the next section, start with the following

Definition 3.1. A quadratic form $Q: H \rightarrow \mathbf{R}$ has Property $(\dagger)$ if

1. $Q: H \rightarrow \mathbf{R}$ is not identically zero and differentiable

2. there is $\varepsilon>0$ such that $Q()-.\varepsilon\|.\|^{2}$ is weakly lower semi-continuous.

Lemma 3.2. Let $X$ be a subspace of $H$ with the property that: there exists $v_{0}$ with $\left\|v_{0}\right\|=1$ in $X$ such that $Q\left(v_{0}\right)=\inf \{Q(v): v \in X,\|v\|=1\}$. Then

$$
\hat{Q}\left(v_{0}, v\right)=\hat{Q}\left(v_{0}, v_{0}\right)<v_{0}, v>
$$

for all $v$ in $X$. 
Proof. Since $v_{0}$ is a critical point for $Q$ on the unit sphere of $X$ there is Lagrange multiplier $\mu$ such that

$$
<(\nabla Q)_{v_{0}}, w>=\mu<(\nabla\|\cdot\|)_{v_{0}}, w>
$$

or equivalently

$$
\hat{Q}\left(v_{0}, w\right)=\mu<v_{0}, w>
$$

for all $w$ in $X$. Now set $w=v_{0}$.

The following Proposition shows that if $Q$ is non-negative then Property $(\dagger)$ guarantees that 0 is at most an isolated point of its spectrum. (The Yang-Mills-Higgs Hessian at the spherically symmetric solution at $\lambda=0$ is non-negative and satisfies $(\dagger)$, see Section 4.)

Proposition 3.3. Any $Q$ satisfying $Q(v) \geq 0$ for all $v$ in $H$ and Property ( $\dagger)$ also satisfies

$$
\inf \{Q(v): v \perp \operatorname{Ker} Q,\|v\|=1\} \supsetneqq 0 .
$$

Proof. Assume to the contrary that

$$
\inf \{Q(v): v \perp \operatorname{Ker} Q,\|v\|=1\}=0 .
$$

By Property $(\dagger),(\operatorname{Ker} Q)^{\perp}$ is non-zero. Choose a sequence $v_{n}$ in $(\operatorname{Ker} Q)^{\perp}$ with $\left\|v_{n}\right\|=1$ such that $Q\left(v_{n}\right) \rightarrow 0$. Passing to a subsequence if necessary, assume that there exists $v_{0}$ in $(\operatorname{Ker} Q)^{\perp}$ such that $v_{n} \rightarrow v_{0}$ weakly in $H$. That $v_{0}$ is not 0 and indeed $\left\|v_{0}\right\|=1$, follows from (2) of Property $(\dagger)$ which implies that

$$
Q\left(v_{0}\right)-\varepsilon\left\|v_{0}\right\|^{2} \leq \liminf \left(Q\left(v_{n}\right)-\varepsilon\left\|v_{n}\right\|^{2}\right)=-\varepsilon .
$$

By (2) of Property $(\dagger), Q$ is also weekly lower semi-continuous. Therefore $Q\left(v_{0}\right)=0$ by the assumption. Now use Lemma 3.2 to have that for any $v$ in $(\operatorname{Ker} Q)^{\perp}$

$$
\hat{Q}\left(v_{0}, v\right)=\hat{Q}\left(v_{0}, v_{0}\right)<v_{0}, v>=0 .
$$

Since also $\hat{Q}\left(v_{0}, v\right)=0$ for $v$ in $\operatorname{Ker} Q, v_{0}$ lies in $\operatorname{Ker} Q$. I.e. $v_{0}$ would have to lie both in $\operatorname{Ker} Q$ and $(\operatorname{Ker} Q)^{\perp}$ and be non-zero. 
Definition 3.4. Let $(H,<,>)$ be a Hilbert space and $V_{\lambda}, V_{0}$ be closed subspaces of $H$ for $\lambda>0$. $V_{\lambda}$ contains $V_{0}$ at the limit as $\lambda \rightarrow 0$ if for all $\varepsilon>0$ there is $\delta>0$ such that $|\lambda-0|<\delta$ implies that for any $u$ in $V_{\lambda}^{\perp}$ of norm 1 there exists $v^{\prime}$ in $V_{0}^{\perp}$ of norm 1 such that $\left\|v^{\prime}-u\right\|<\varepsilon$.

The following shows that the uniform convergence on the unit sphere and the existence of "kernel-subspaces containing kernel at the limit", along with some fairly mild assumptions, guarantee that if zero is an isolated point of the spectrum of $Q_{0}$ then it is an isolated point of the spectrum of $Q_{\lambda}$ for $\lambda$ in an open neighborhood of 0. (The Yang-Mills-Higgs Hessians at spherically symmetric configurations have kernel-subspaces containing kernel at the limit, see Section 4.)

Theorem 3.5. Let $Q_{\lambda}(\lambda>0)$ and $Q_{0}$ be quadratic forms and assume that there are subspaces $N_{\lambda}$ of $\operatorname{Ker} Q_{\lambda}$ satisfying

1. $Q_{0}$ is uniformly continuous on the unit sphere of $H$ i.e. for all $\varepsilon>0$ there exists $\delta>0$ such that if $u, v$ are on the unit sphere of $H$ and $\|u-v\|<\delta$ then $\left|Q_{0}(u)-Q_{0}(v)\right|<\varepsilon$.

2. $\alpha:=\inf \left\{Q_{0}(v): v \perp \operatorname{Ker} Q_{0},\|v\|=1\right\} \supsetneqq 0$

3. $\sup _{\|v\|=1}\left|Q_{0}(v)-Q_{\lambda}(v)\right| \rightarrow 0$ as $\lambda \rightarrow 0$

4. $N_{\lambda}$ contains $\operatorname{Ker} Q_{0}$ at the limit as $\lambda \rightarrow 0$.

Then there exists $\varepsilon>0$ such that whenever $0<\lambda<\varepsilon$ then

1. $\inf \left\{Q_{\lambda}(v): v \perp N_{\lambda},\|v\|=1\right\}>\frac{\alpha}{3} \supsetneqq 0$

2. $N_{\lambda}=\operatorname{Ker} Q_{\lambda}$.

Proof. Fix $u$ in $N_{\lambda}^{\perp}$ with $\|u\|=1$, for $\left|\lambda-\lambda_{0}\right|<\eta$, where $\eta$ is guaranteed by (3) to give $\left|Q_{0}(u)-Q_{\lambda}(u)\right|<\alpha / 3$. Therefore

$$
Q_{\lambda}(u) \geq Q_{0}(u)-\left|Q_{0}(u)-Q_{\lambda}(u)\right|>Q_{0}(u)-\alpha / 3 .
$$

Now use Definition 3.4 and (4) to choose $\eta^{\prime}$ such that $0<\lambda<\eta^{\prime}$ gives a $v$ of norm 1 in $\left(\operatorname{Ker} Q_{0}\right)^{\perp}$ such that $\|v-u\|<\delta$, for the $\delta$ guaranteed by (1) for $\varepsilon=\alpha / 3$. Then $0<\lambda<\min \left\{\eta, \eta^{\prime}\right\}$ implies that

$$
Q_{\lambda}(u) \geq Q_{0}(v)-2 \alpha / 3 \geq a / 3>0
$$


by $(2)$.

To see that $N_{\lambda}=\operatorname{Ker} Q_{\lambda}$, merely note that $H=N_{\lambda}+\left(N_{\lambda}\right)^{\perp}$.

\section{Application to the Yang-Mills-Higgs Hessians}

This section will demonstrate that the conditions required for the results of the previous sections hold for Yang-Mills-Higgs hessians.

Start by recalling that as in [T2], the Yang-Mills-Higgs Hessian for $\lambda=0$ can be written as

$$
Q_{0}^{c}(a, \phi)=\|(a, \phi)\|_{c}^{2}+2<F_{A},[a, a]>+4<d_{A} \Phi,[a, \phi]>
$$

where $<,>$ is the $L^{2}$ inner product on $\mathbf{R}^{3}$.

Proposition 4.1. For any $c$ in $\mathcal{C}$ the corresponding Hessian $Q_{0}^{c}$ satisfies the following:

1. $Q_{0}^{c}: T_{c} \mathcal{C} \rightarrow \mathbf{R}$ is differentiable and uniformly continuous on the unit ball of $\left(T_{c} \mathcal{C},\|\cdot\|_{c}\right)$.

2. there is $\varepsilon>0$ such that $Q_{0}^{c}-\varepsilon\|.\|_{c}^{2}$ is weakly lower semi-continuous.

Proof. (1) For any quadratic form $Q$ its differential at $v$ is $D Q_{v}(h)=\hat{Q}(h, v)+\hat{Q}(v, h)$ whenever $Q$ is continuous at 0 . The continuity of the quadratic form at hand follows from equation (5) and from the estimates

$$
\begin{gathered}
<F_{A},[a, a]>\leq\left\|F_{A}\right\|_{2}\|[a, a]\|_{2} \leq C\left\|F_{A}\right\|_{2}\|(a, \phi)\|_{c}^{2}, \\
<d_{A} \Phi,[a, \phi]>\leq\left\|d_{A} \Phi\right\|_{2}\|[a, \phi]\|_{2} \leq C\left\|D_{A} \Phi\right\|_{2}\|(a, \phi)\|_{c}^{2} .
\end{gathered}
$$

For the proof that

$$
\|[a, a]\|_{2} \leq C\|(a, \phi)\|_{c}^{2}, \quad\|[a, \phi]\|_{2} \leq C\|(a, \phi)\|_{c}^{2}
$$

see Lemma B6.4 of [T2]. The proof of the uniform continuity follows along similar lines and is left to the reader.

For (2), write the Hessian as in (5) so that

$$
Q_{0}^{c}(a, \phi)-\varepsilon\|(a, \phi)\|_{c}^{2}=(1-\varepsilon)\|(a, \phi)\|_{c}^{2}+2<F_{A},[a, a]>+4<d_{A} \Phi,[a, \phi]>
$$


For $\varepsilon<1$ the first term in the right hand side is clearly weakly lower semi-continuous. For the remaining terms, following section VI. of [T2], note that the finite energy of $(A, \Phi)$ and the estimates of part (1) of this Proposition imply that for any $\delta>0$ there exists $r>0$ such that on the complement of the ball of radius $r$ the following are true:

$$
\begin{aligned}
& <F_{A},[a, a]>\leq \delta\|(a, \phi)\|_{c}^{2}, \\
& <d_{A} \Phi,[a, \phi]>\leq \delta\|(a, \phi)\|_{c}^{2} .
\end{aligned}
$$

From these it is standard to show that the last two terms of (6) are weakly continuous.

Now to employ the results of the previous section as a sequence of configurations $c_{i}$ converges to $c_{0}$ in $\mathcal{C}$ it is convenient to realize all the quadratic forms involved on the same Hilbert space. Happily, this is precisely the context of Propositions B6.1. and B6.2. of [T2]: for $c$ and $c_{0}$ sufficiently close in $\mathcal{C}$ the identity map on $C_{0}^{\infty}\left(\mathbf{R}^{3}\right)$ induces an isomorphism between the tangent spaces $\left(T_{c},\|\cdot\|_{c}\right)$ and $\left(T_{c_{0}},\|\cdot\|_{c_{0}}\right)$. Furthermore, a neighborhood of $c_{0}$ in $\mathcal{C}$ can be identified with a ball around the origin of $T_{c_{0}}$, so that $c$ is close to $c_{0}$ if $c-c_{0}$ has small $T_{c_{0}}$-norm.

With this understood, and after equipping all $T_{c}$ 's for $c$ in a sufficiently small neighborhood of $c_{0}$ in $\mathcal{C}$ with the $c_{0}$-inner product, it makes sense to compare the Hessians using the $c_{0^{-}}$ norm:

Lemma 4.2. Let $c_{i}$ converge to $c_{0}$ in $\mathcal{C}$. Then $\sup _{\|v\|_{c_{0}}=1}\left|Q_{0}^{c_{i}}(v)-Q_{0}^{c_{0}}(v)\right| \rightarrow 0$.

Proof. Simply calculate that for some constant $C$ depending only on $c_{0}$

$$
\left|Q_{0}^{c_{i}}(v)-Q_{0}^{c_{0}}(v)\right| \leq C\|v\|_{c_{0}}^{2}\left\|c_{i}-c_{0}\right\|_{c_{0}} .
$$

(This is statement (5) of Proposition A.4.3. of [T3].)

When $\lambda$ is not zero the Hessian $Q_{\lambda}$ will be defined on $T \mathcal{C}_{+}$rather than the whole of $T \mathcal{C}$. The following shows that, due to the rather remarkable fact that the only part of $\phi$ that matters for the $\lambda$-terms of $Q_{\lambda}$ is the part that is orthogonal to $\Phi$ in the Lie Algebra, the $c_{0}$-norm suffices to control the $Q_{\lambda}$ Hessian from below even when $\lambda \neq 0$. 
Lemma 4.3. Assume that $\lambda \rightarrow 0$, that $\left|\Phi_{\lambda}\right|(x)<1$ for all $x$ and that there is $R$ such that for all $\lambda$ smaller than some $\lambda_{0}$ and $x$ with $|x|>R, \frac{1}{2}<\left|\Phi_{\lambda}\right|^{2}(x)$.

Then

$$
\liminf _{\lambda \rightarrow 0} \inf _{u \in T \mathcal{C}_{+},\|u\|_{c_{0}}=1}\left(Q_{\lambda}^{c_{\lambda}}(u)-Q_{0}^{c_{\lambda}}(u)\right) \geq 0
$$

Proof. First note that for $|x|>R$

$$
\frac{1}{2}|\phi|^{2}<\left|\Phi_{\lambda}\right|^{2}|\phi|^{2}=<\Phi_{\lambda}, \phi>^{2}+\left|\left[\Phi_{\lambda}, \phi\right]\right|^{2} .
$$

Now for $u=(a, \phi)$ with $\|u\|_{c_{0}}=1$ estimate as follows:

$$
\begin{aligned}
\frac{\lambda}{2} \int_{\mathbf{R}^{3}} & \left(\left|\Phi_{\lambda}\right|^{2}-1\right)|\phi|^{2} d^{3} x+\lambda \int_{\mathbf{R}^{3}}<\Phi_{\lambda}, \phi>^{2} d^{3} x \\
\geq & \frac{\lambda}{2} \int_{|x| \leq R}\left(\left|\Phi_{\lambda}\right|^{2}-1\right)|\phi|^{2} d^{3} x+\frac{\lambda}{2} \int_{|x|>R}\left(\left|\Phi_{\lambda}\right|^{2}-1\right)|\phi|^{2} d^{3} x+\lambda \int_{|x|>R}<\Phi_{\lambda}, \phi>^{2} d^{3} x \\
\geq & -\frac{\lambda}{2} \int_{|x| \leq R}|\phi|^{2} d^{3} x+\frac{\lambda}{2} \int_{|x|>R}\left(\left|\Phi_{\lambda}\right|^{2}-1\right) 2\left(\left|\left[\Phi_{\lambda}, \phi\right]\right|^{2}+<\Phi_{\lambda}, \phi>^{2}\right) d^{3} x \\
& \quad+\lambda \int_{|x|>R}<\Phi_{\lambda}, \phi>^{2} d^{3} x \\
\geq & -\frac{\lambda}{2} C_{R}\|\phi\|_{6}^{2}+\lambda \int_{|x|>R}\left(\left|\Phi_{\lambda}\right|^{2}-1\right)\left|\left[\Phi_{\lambda}, \phi\right]\right|^{2} d^{3} x+\lambda \int_{|x|>R}\left|\Phi_{\lambda}\right|^{2}<\Phi_{\lambda}, \phi>^{2} d^{3} x \\
\geq & -\lambda\left(\frac{1}{2} C_{R}+1\right)\|u\|_{c_{0}}^{2},
\end{aligned}
$$

where $C_{R}$ is a constant depending only on $R$.

Now Proposition 4.1, Lemmata 4.2 and 4.3 together with Theorem 3.5 yield the following:

Theorem 4.4. Assume that

1. As $\lambda \rightarrow 0, c_{\lambda}$ converge to $c_{0}$ in $\mathcal{C}$

2. $Q_{0}^{c_{0}}$ is non-negative and not identically 0 .

3. $\left|\Phi_{\lambda}\right|(x)<1$ for all $x$ and there is $V$ compact in $\mathbf{R}^{3}$ such that for all $\lambda$ sufficiently small and $x$ not in $V, \frac{1}{2}<\left|\Phi_{\lambda}\right|^{2}(x)$.

4. there are subspaces $N_{\lambda}$ of $\operatorname{Ker} Q_{0}^{c_{\lambda}}$ containing $\operatorname{Ker} Q_{0}$ at the limit

Then for $\lambda$ small enough

$$
\operatorname{Ker} Q_{\lambda}^{c_{\lambda}}=N_{\lambda}
$$


and

$$
Q_{\lambda}^{c_{\lambda}} \geq 0
$$

Proof. By assumption 2 and Proposition 4.1, $Q_{0}^{c_{0}}$ has property $(\dagger)$; therefore by Proposition 3.3 is strictly positive away from its kernel.

Assumption 1 and Lemma 4.2 give

$$
\sup _{\|v\|_{c_{0}=1}}\left|Q_{0}^{c_{\lambda}}(v)-Q_{0}^{c_{0}}(v)\right| \rightarrow 0
$$

as $\lambda \rightarrow 0$. With this and the first part of Lemma 4.1, all assumptions of Theorem 3.5 are satisfied, therefore there is $I>0$ such that for sufficiently small $\lambda$

$$
\inf \left\{Q_{0}^{c_{\lambda}}(v):\|v\|_{c_{0}}=1, v \perp N_{\lambda}\right\} \geq I
$$

and

$$
N_{\lambda}=\operatorname{Ker} Q_{0}^{c_{\lambda}}
$$

By assumption 3 and Lemma 4.3

$$
\inf \left\{Q_{\lambda}^{c_{\lambda}}(v): v \in T \mathcal{C}_{+},\|v\|_{c_{0}}=1, v \perp N_{\lambda}\right\} \geq I / 2
$$

for small $\lambda$. For those $\lambda$,

$$
Q_{\lambda}^{c_{\lambda}} \geq 0
$$

and

$$
\operatorname{Ker} Q_{\lambda}^{c_{\lambda}}=\operatorname{Ker} Q_{0}^{c_{\lambda}}=N_{\lambda} .
$$

(since $N_{\lambda} \subset \operatorname{Ker} Q_{\lambda}^{c_{\lambda}}$ and $N_{\lambda}^{\perp} \cap \operatorname{Ker} Q_{\lambda}^{c_{\lambda}}=\{0\}$ ).

\section{ApPliCATION TO THE SPHERICALly SYMMETRIC MONOPOLE SOLUTIONS}

This section argues that the conditions of Theorem 4.4 are satisfied when $c_{\lambda}$ is the sequence of spherically symmetric solutions of the variational equations for $E_{\lambda}$.

In $[\mathrm{tH}]$ and $[\mathrm{P}]$, 't Hooft and Polyakov suggested spherically symmetric solutions for the three-dimensional Yang-Mills-Higgs equations. With respect to the standard basis $e_{a}, a=$ $1,2,3$ of $\mathfrak{s u}(2)$ their Ansatz reads

$$
A=\varepsilon_{i j a} \frac{x_{j}}{r^{2}}(1-K(r)) e_{a} d x_{i}
$$




$$
\Phi=\frac{x_{\alpha}}{r} \frac{H(r)}{r} e_{a} .
$$

When evaluated on configurations of this form, the Yang-Mills-Higgs functional with selfinteraction parameter $\lambda$ reduces to the one-dimensional integral

$$
\begin{aligned}
E_{\lambda}(H, K)=4 \pi \int_{0}^{\infty}\left\{\left(K^{\prime}\right)^{2}\right. & +\frac{1}{2}\left(H^{\prime}-\frac{H}{r}\right)^{2}+\frac{K^{2} H^{2}}{r^{2}}+\frac{1}{2} \frac{\left(K^{2}-1\right)^{2}}{r^{2}} \\
& \left.+\frac{\lambda}{4}\left(\frac{H^{2}}{r}-r\right)^{2}\right\} d r .
\end{aligned}
$$

Further assume the boundary conditions

$$
\begin{gathered}
K(r) \rightarrow 0, \quad r \rightarrow \infty, \\
\frac{H}{r} \rightarrow 1, \quad r \rightarrow \infty,
\end{gathered}
$$

which, although stronger than finite action, are satisfied by any finite action critical point for $\lambda>0$, see $[\mathrm{M}]$. Therefore for $\lambda>0$ there is no loss of generality under these conditions. For $\lambda=0$ the asymptotic behavior of $\frac{H}{r}$ is ad hoc.

At a critical point $\left(K_{\lambda}, H_{\lambda}\right)$ of $E_{\lambda}$, the variational equation

$$
\left.\frac{d}{d t}\right|_{t=0} E_{\lambda}\left(H_{\lambda}+t h, K_{\lambda}+t k\right)=0
$$

for all $h, k$ of compact support, yields the following system of non-linear, second order, ordinary differential equations

$$
\begin{aligned}
& K_{\lambda}^{\prime \prime}=\frac{H_{\lambda}^{2}-1+K_{\lambda}^{2}}{r^{2}} K_{\lambda} \\
& H_{\lambda}^{\prime \prime}=\frac{2 K_{\lambda}^{2}}{r^{2}} H_{\lambda}-4 \lambda H_{\lambda}\left(1-\frac{H_{\lambda}^{2}}{r^{2}}\right)
\end{aligned}
$$

The 't Hooft-Polyakov Ansatz has fixed gauge so that solutions are smooth.

For rigorous proofs (of various degrees of generality) of the existence of such spherically symmetric solutions for any $\lambda \geq 0$, see [TFS], [RFS], [R] and [D2]. What is important to recall at this point is that for each $\lambda$, the 't Hooft-Polyakov solution is obtained by minimizing 
$E_{\lambda}$ over all $(K, H)$ of the form above. For the remainder of this paper, and for $\lambda \geq 0$,

$$
c_{\lambda}=\left(K_{\lambda}, H_{\lambda}\right)
$$

will denote this solution.

Crucial in what follows is the explicit solution of (YMH 1-2) for $\lambda=0$, found by Prasad and Sommerfield:

$$
\begin{gathered}
H_{0}=r \operatorname{coth} r-1, \\
K_{0}=\frac{r}{\sinh r} .
\end{gathered}
$$

That the 't Hooft-Polyakov spherically symmetric solution $c_{0}$ is precisely this Prasad- Sommerfiled solution was proved in $[\mathrm{M}]$. Note that although $E_{0}\left(c_{0}\right)$ is finite, $E_{\lambda}\left(c_{0}\right)$ is not finite for $\lambda>0$.

Definition 5.1. For $c_{\lambda}=\left(A_{\lambda}, \Phi_{\lambda}\right)$ the 3-dimensional subspace $S_{c_{\lambda}}$ of $T_{c} \mathcal{C}$ is defined as the linear span

$$
S_{\lambda}=\left\langle\left(\frac{\partial A_{\lambda}}{\partial x_{\alpha}}, \frac{\partial \Phi_{\lambda}}{\partial x_{\alpha}}\right), \alpha=1,2,3\right\rangle .
$$

Observe that for each $\lambda \geq 0$ the space $S_{\lambda}$ is always in the Kernel of $Q_{\lambda}^{c_{\lambda}}$ and of $Q_{0}^{c_{\lambda}}$. This follows from the fact that the energy functional for any value of $\lambda$ is invariant under translations. By finite energy the translates cannot be gauge equivalent to each other, hence they are non-zero vectors in the tangent space. A straightforward calculation shows that the three vectors are linearly independent.

To identify the Kernel of $Q_{0}^{c_{0}}$ it is useful to rewrite the Hessian yet again:

\section{Lemma 5.2.}

$$
Q_{0}^{c_{0}}(a, \phi)=\left\|* d_{A} a-d_{A} \phi-[a, \Phi]\right\|_{2}^{2}+<* F_{A}-d_{A} \Phi, *[a, a]-[a, \phi]>_{2} .
$$

Proof. Recall that the magnetic charge

$$
N=\frac{1}{4 \pi} \int_{\mathbf{R}^{3}} \operatorname{tr}\left(F_{A} \wedge d_{A} \Phi\right)
$$

is a constant on each path component of $\mathcal{C}$ and differentiate it twice to get the identity

$$
2<* F_{A},[a, \phi]>+<* d_{A} \Phi,[a, a]>+2<d_{A} \phi+[a, \Phi], * d_{A} a>=0 .
$$


Therefore,

$$
\begin{aligned}
Q_{0}^{c_{0}}(a, \phi) & =\left\|d_{A} \phi+[a, \Phi]\right\|^{2}+\left\|d_{A} a\right\|^{2}+<F_{A},[a, a]>+2<d_{A} \Phi,[a, \phi]> \\
& =\left\|d_{A} \phi+[a, \Phi]\right\|^{2}+\left\|d_{A} a\right\|^{2}+<F_{A}-* d_{A} \Phi,[a, a]> \\
& +<* d_{A} \Phi,[a, a]>+2<d_{A} \Phi-* F_{A},[a, \phi]>+2<* F_{A},[a, \phi]>.
\end{aligned}
$$

Now use equation (9) to replace the last and third-from-the-end terms in the right hand side by

$$
-2<d_{A} \phi+[a, \Phi], * d_{A} a>
$$

to get

$$
\begin{aligned}
Q_{0}^{c_{0}}(a, \phi) & =\left\|d_{A} \phi+[a, \Phi]\right\|^{2}+\left\|d_{A} a\right\|^{2}+<F_{A}-* d_{A} \Phi,[a, a]> \\
& -2<d_{A} \phi+[a, \Phi], * d_{A} a>+2<d_{A} \Phi-* F_{A},[a, \phi]> \\
& =\left\|d_{A} \phi+[a, \Phi]-* d_{A} a\right\|^{2}+<F_{A}-* d_{A} \Phi,[a, a]-2 *[a, \phi]>.
\end{aligned}
$$

Proposition 5.3. $\operatorname{Ker}\left(Q_{0}^{c_{0}}\right)=S_{0}$.

Proof. By $[\mathrm{M}]$, the spherically symmetric solution $\left(A_{0}, \Phi_{0}\right)$ solves the first order Bogomol'nyi equation (2), therefore the second term of $Q_{0}^{c_{0}}$ when rewritten as in Lemma 5.2 vanishes. Then $Q_{0}^{c_{0}}(a, \phi)$ is zero if and only if

$$
* d_{A} a-d_{A} \phi-[a, \Phi]=0
$$

But this is exactly the linearization of equation (2), i.e. the only zero directions are the directions tangent to the moduli space of charge 1 minima. With the conventions of section 2 the main result in $[\mathrm{M}]$ gives that the dimension of this moduli space is 3 . (The dimension counting there gives an extra dimension that has been excluded here by the choice of norm on $T_{c} \mathcal{C}$.) Since the energy is invariant under translations, all translates of a minimum are still minima and this accounts for all minima. Therefore $Q_{0}^{c_{0}}$ is zero only on the translation directions.

Propositions 4.1 and 5.3 together yield 
Corollary 5.4. The Yang-Mills-Higgs Hessian $Q_{0}^{c_{0}}$ at the spherically symmetric solution for $\lambda=0$ has Property $(\dagger)$.

The remaining sections of this paper will be devoted to proving the following

Theorem 5.5. $\quad$ 1. The $\lambda$-spherically symmetric solution $c_{\lambda}$ converges to $c_{0}$ in $\mathcal{C}$ as $\lambda \rightarrow 0$.

2. $\left|\Phi_{\lambda}\right|(x)<1$ for all $x$ and there is $V$ compact in $\mathbf{R}^{3}$ such that for $\lambda$ sufficiently small and $x$ not in $V, \frac{1}{2}<\left|\Phi_{\lambda}\right|^{2}(x)$.

3. $S_{\lambda}$ contain $\operatorname{Ker} Q_{0}^{c_{0}}$ at the limit.

With this, all conditions are satisfied for Theorem 4.4 to yield

Theorem 5.6. For $\lambda$ in an neighborhood of 0 , the Hessian of the $\lambda$-'t Hooft-Polyakov monopole satisfies

$$
Q_{\lambda}^{c_{\lambda}}(a, \phi) \geq 0
$$

for any $(a, \phi)$ in $H_{\lambda}$.

Furthermore,

$$
\operatorname{Ker}\left(Q_{\lambda}^{c_{\lambda}}\right)=S_{\lambda}
$$

Corollary 5.7. There is $\lambda_{0}$ such that for $\lambda \leq \lambda_{0}$, the Yang-Mills-Higgs functional $E_{\lambda}$ has no decreasing directions at $\left(A_{\lambda}, \Phi_{\lambda}\right)$.

Proof. Use equation (3).

\section{Outline of proof of Theorem 5.5}

Proof of Part 1. of Theorem 5.5

According to the definition of the topology on $\mathcal{C}$, the following are needed:

I. On any $K$ compact in $\mathbf{R}^{3}$,

$$
\left\|c_{\lambda}-c_{0}\right\|_{L_{1}^{2}(K)} \rightarrow 0
$$

\section{On $\mathbf{R}^{3}$}

$$
\left\|F_{A_{\lambda}}\right\|_{2}-\left\|F_{A_{0}}\right\|_{2} \rightarrow 0
$$




\section{On $\mathbf{R}^{3}$}

$$
\left\|d_{A_{\lambda}} \Phi_{\lambda}\right\|_{2}-\left\|d_{A_{0}} \Phi_{0}\right\|_{2} \rightarrow 0
$$

I. follows for any compact $K$ if it is true on any sphere centered at 0 of radius $b$. For this, calculate

$$
\begin{aligned}
\left\|A_{\lambda}-A_{0}\right\|_{L^{2}(K)}^{2}+\left\|\nabla\left(A_{\lambda}-A_{0}\right)\right\|_{L^{2}(K)}^{2} \leq & C\left(\int_{0}^{b}\left(K_{\lambda}-K_{0}\right)^{2} d r+\int_{0}^{b}\left(K_{\lambda}^{\prime}-K_{0}^{\prime}\right)^{2} d r\right. \\
& \left.+\int_{0}^{b} \frac{1}{r^{2}}\left(K_{\lambda}-K_{0}\right)^{2} d r\right)
\end{aligned}
$$

and

$$
\begin{aligned}
\left\|\Phi_{\lambda}-\Phi_{0}\right\|_{L^{2}(K)}^{2}+\left\|\nabla\left(\Phi_{\lambda}-\Phi_{0}\right)\right\|_{L^{2}(K)}^{2} & \leq C\left(\int_{0}^{b}\left(H_{\lambda}-H_{0}\right)^{2} d r+\int_{0}^{b}\left(H_{\lambda}^{\prime}-H_{0}^{\prime}\right)^{2} d r\right. \\
& \left.+\int_{0}^{b} \frac{1}{r^{2}}\left(H_{\lambda}-H_{0}\right)^{2} d r\right)
\end{aligned}
$$

That the right-hand side of (10) goes to zero as $\lambda$ goes to 0 is part of Theorem 11.2.

That the right-hand side of (11) goes to zero as $\lambda$ goes to 0 is part of Theorem 11.1.

II. and III. follow from the fact that for small $\lambda$

$$
\left|\left\|F_{A_{\lambda}}\right\|_{2}-\left\|F_{A_{0}}\right\|_{2}\right|+\left|\left\|d_{A_{\lambda}} \Phi_{\lambda}\right\|_{2}-\left\|d_{A_{0}} \Phi_{0}\right\|_{2}\right| \leq C\left\|c_{\lambda}-c_{0}\right\|_{c_{0}}
$$

see part (1) of Proposition A.4.3. of [T4]. To estimate the right-hand side of this, calculate

$$
\begin{aligned}
& \left\|c_{\lambda}-c_{0}\right\|_{c_{0}}^{2}=\left\|\nabla_{A_{0}}\left(A_{\lambda}-A_{0}\right)\right\|_{2}^{2}+\left\|\nabla_{A_{0}}\left(\Phi_{\lambda}-\Phi_{0}\right)\right\|_{2}^{2} \\
& +\left\|\left[\Phi_{0}, A_{\lambda}-A_{0}\right]\right\|_{2}^{2}+\left\|\left[\Phi_{0}, \Phi_{\lambda}-\Phi_{0}\right]\right\|_{2}^{2} \\
& \leq\left\|\nabla\left(A_{\lambda}-A_{0}\right)\right\|_{2}^{2}+\left\|\left[A_{0}, A_{\lambda}-A_{0}\right] \quad\right\|_{2}^{2} \\
& +\left\|\nabla\left(\Phi_{\lambda}-\Phi_{0}\right)\right\|_{2}^{2}+\left\|\quad\left[A_{0}, \Phi_{\lambda}-\Phi_{0}\right] \quad\right\|_{2}^{2} \\
& +\left\|\left[\Phi_{0}, A_{\lambda}-A_{0}\right] \quad\right\|_{2}^{2}+\left\|\left[\Phi_{0}, \Phi_{\lambda}-\Phi_{0}\right] \quad\right\|_{2}^{2} .
\end{aligned}
$$

The right hand side of this is bounded as follows:

$$
\begin{gathered}
\left\|\nabla\left(A_{\lambda}-A_{0}\right)\right\|_{2}^{2} \leq \int_{0}^{\infty}\left(K_{\lambda}^{\prime}-K_{0}^{\prime}\right)^{2}+\frac{1}{r^{2}}\left(K_{\lambda}-K_{0}\right)^{2} d r \\
\left\|\quad\left[A_{0}, A_{\lambda}-A_{0}\right] \quad\right\|_{2}^{2} \leq \max _{\mathbf{R}^{3}}\left|A_{0}\right|\left\|A_{\lambda}-A_{0}\right\|_{2}^{2} \leq C \int_{0}^{\infty}\left(K_{\lambda}-K_{0}\right)^{2} d r,
\end{gathered}
$$




$$
\begin{gathered}
\left\|\nabla\left(\Phi_{\lambda}-\Phi_{0}\right)\right\|_{2}^{2} \leq \int_{0}^{\infty}\left(H_{\lambda}^{\prime}-H_{0}^{\prime}\right)^{2}+\frac{1}{r^{2}}\left(H_{\lambda}-H_{0}\right)^{2} d r \\
\left\|\quad\left[A_{0}, \Phi_{\lambda}-\Phi_{0}\right]\right\|_{2}^{2} \leq\left\|\left|A_{0}\right|\left|\Phi_{\lambda}-\Phi_{0}\right|\right\|_{2}^{2} \\
\leq C\left\|\frac{1}{r}\left|\Phi_{\lambda}-\Phi_{0}\right|\right\|_{2}^{2} \\
\leq C \int_{0}^{\infty} \frac{1}{r^{2}}\left(H_{\lambda}-H_{0}\right)^{2} d r \\
\left\|\quad\left[\Phi_{0}, A_{\lambda}-A_{0}\right] \quad\right\|_{2}^{2} \leq\left\|A_{\lambda}-A_{0}\right\|_{2}^{2} \leq C \int_{0}^{\infty}\left(K_{\lambda}-K_{0}\right)^{2} d r, \\
{\left[\Phi_{0}, \Phi_{\lambda}-\Phi_{0}\right]=0 .}
\end{gathered}
$$

That the right hand sides of all the above inequalities go to 0 as $\lambda$ tends to 0 , is proved as Theorems 11.2 and 11.1 .

Proof of Part 2. of Theorem 5.5

This follows from Theorem IV.10.1 of [JT] and Lemma 7.2 below.

Proof of Part 3. of Theorem 5.5

This follows immediately from

Theorem 6.1. As $\lambda \rightarrow 0$,

$$
\left\|\left(\frac{\partial A_{\lambda}}{\partial x_{a}}, \frac{\partial \Phi_{\lambda}}{\partial x_{a}}\right)-\left(\frac{\partial A_{0}}{\partial x_{a}}, \frac{\partial \Phi_{0}}{\partial x_{a}}\right)\right\|_{c_{0}} \rightarrow 0 .
$$

\section{Proof of Theorem 6.1}

As a matter of a straightforward calculation using that $\left|\Phi_{0}\right|(x)<1$, and that $\left|A_{0}\right|(x)<1$,

$$
\begin{aligned}
\left\|\frac{\partial c_{\lambda}}{\partial x_{a}}-\frac{\partial c_{0}}{\partial x_{a}}\right\|_{c_{0}}^{2}= & \left\|\nabla_{A_{0}} \frac{\partial\left(A_{\lambda}-A_{0}\right)}{\partial x_{a}}\right\|_{2}^{2}+\left\|\nabla_{A_{0}} \frac{\partial\left(\Phi_{\lambda}-\Phi_{0}\right)}{\partial x_{a}}\right\|_{2}^{2} \\
& +\left\|\left[\Phi_{0}, \frac{\partial\left(A_{\lambda}-A_{0}\right)}{\partial x_{a}}\right]\right\|_{2}^{2}+\left\|\left[\Phi_{0}, \frac{\partial\left(\Phi_{\lambda}-\Phi_{0}\right)}{\partial x_{a}}\right] \quad\right\|_{2}^{2} \\
\leq & C\left(\left\|\nabla \frac{\partial\left(A_{\lambda}-A_{0}\right)}{\partial x_{a}}\right\|_{2}^{2}+\left\|\nabla \frac{\partial\left(\Phi_{\lambda}-\Phi_{0}\right)}{\partial x_{a}}\right\|_{2}^{2}\right. \\
& \left.+\left\|\frac{\partial\left(A_{\lambda}-A_{0}\right)}{\partial x_{a}}\right\|_{2}^{2}+\left\|\frac{\partial\left(\Phi_{\lambda}-\Phi_{0}\right)}{\partial x_{a}}\right\|_{2}^{2}\right) .
\end{aligned}
$$


Now calculate that

$$
\begin{aligned}
\left\|\nabla \frac{\partial\left(A_{\lambda}-A_{0}\right)}{\partial x_{a}}\right\|_{2}^{2} \leq & C\left(\int_{0}^{\infty} \frac{1}{r^{4}}\left(K_{0}-K_{\lambda}\right)^{2} d r\right. \\
& +\int_{0}^{\infty} \frac{1}{r^{2}}\left(K_{0}^{\prime}-K_{\lambda}^{\prime}\right)^{2} d r \\
& \left.+\int_{0}^{\infty}\left(K_{0}^{\prime \prime}-K_{\lambda}^{\prime \prime}\right)^{2} d r\right)
\end{aligned}
$$

That the right hand side of this inequality goes to zero is part of Theorem 11.2.

Also,

$$
\begin{aligned}
\left\|\nabla \frac{\partial\left(\Phi_{\lambda}-\Phi_{0}\right)}{\partial x_{a}}\right\|_{2}^{2} \leq & C\left(\int_{0}^{\infty} \frac{1}{r^{4}}\left(H_{0}-H_{\lambda}\right)^{2} d r\right. \\
& +\int_{0}^{\infty} \frac{1}{r^{2}}\left(H_{0}^{\prime}-H_{\lambda}^{\prime}\right)^{2} d r \\
& \left.+\int_{0}^{\infty}\left(H_{0}^{\prime \prime}-H_{\lambda}^{\prime \prime}\right)^{2} d r\right) .
\end{aligned}
$$

That the right hand side of this inequality goes to zero as $\lambda$ goes to zero is part of Theorem 11.1. Similarly,

$$
\begin{aligned}
\left\|\frac{\partial\left(A_{\lambda}-A_{0}\right)}{\partial x_{a}}\right\|_{2}^{2} \leq C\left(\int_{0}^{\infty} \frac{1}{r^{2}}\left(K_{0}-K_{\lambda}\right)^{2} d r+\int_{0}^{\infty}\left(K_{0}^{\prime}-K_{\lambda}^{\prime}\right)^{2} d r\right), \\
\left\|\frac{\partial\left(\Phi_{\lambda}-\Phi_{0}\right)}{\partial x_{a}}\right\|_{2}^{2} \leq C\left(\int_{0}^{\infty} \frac{1}{r^{2}}\left(H_{0}-H_{\lambda}\right)^{2} d r+\int_{0}^{\infty}\left(H_{0}^{\prime}-H_{\lambda}^{\prime}\right)^{2} d r\right) .
\end{aligned}
$$

That the right hand side of these inequalities goes to zero as $\lambda$ goes to zero is proved as Theorems 11.2 and 11.1, respectively.

\section{UNIFORM BOUNDS ON THE ENERGY}

As usual, the first step in the convergence argument for Part 1. of Theorem 5.5 is to show that the part of the energy containing the derivatives of the fields stays uniformly bounded. This is shown in Proposition 7.1 below.

That bounds on the energy imply bounds on derivatives and therefore local convergence is shown as Proposition 7.4 which follows closely arguments in [R]. In this way, Proposition 7.4 is the one-dimensional version of the Uhlenbeck compactness for Yang-Mills-Higgs as in section V. of [T3]. 
Proposition 7.1. Let $c_{\lambda}$ and $c_{\lambda^{\prime}}$ be 't Hooft-Polyakov solutions for $\lambda$ and $\lambda^{\prime}$ respectively, with $\lambda^{\prime}<\lambda$. Then

$$
E_{0}\left(c_{\lambda^{\prime}}\right) \leq E_{0}\left(c_{\lambda}\right)
$$

Proof. First write the functional $E_{\lambda}$ as

$$
E_{\lambda}(c)=E_{0}(c)+\lambda V(c) .
$$

Recall that $c_{\lambda}$ minimizes $E_{\lambda}$ amongst all spherically symmetric configurations. Therefore

$$
E_{\lambda}\left(c_{\lambda}\right) \leq E_{\lambda}\left(c_{\lambda^{\prime}}\right)
$$

or

$$
E_{0}\left(c_{\lambda}\right)+\lambda V\left(c_{\lambda}\right) \leq E_{0}\left(c_{\lambda^{\prime}}\right)+\lambda V\left(c_{\lambda^{\prime}}\right) .
$$

Similarly, $c_{\lambda^{\prime}}$ minimizes $E_{\lambda^{\prime}}$, therefore

$$
E_{\lambda^{\prime}}\left(c_{\lambda^{\prime}}\right) \leq E_{\lambda^{\prime}}\left(c_{\lambda}\right)
$$

or

$$
E_{0}\left(c_{\lambda^{\prime}}\right)+\lambda^{\prime} V\left(c_{\lambda^{\prime}}\right) \leq E_{0}\left(c_{\lambda}\right)+\lambda^{\prime} V\left(c_{\lambda}\right) .
$$

Adding equations (12) and (13)

$$
\lambda V\left(c_{\lambda}\right)+\lambda^{\prime} V\left(c_{\lambda^{\prime}}\right) \leq \lambda V\left(c_{\lambda^{\prime}}\right)+\lambda^{\prime} V\left(c_{\lambda}\right),
$$

or

$$
\left(\lambda-\lambda^{\prime}\right) V\left(c_{\lambda}\right) \leq\left(\lambda-\lambda^{\prime}\right) V\left(c_{\lambda^{\prime}}\right)
$$

Since $\lambda^{\prime}<\lambda$

$$
V\left(c_{\lambda}\right) \leq V\left(c_{\lambda^{\prime}}\right)
$$

This and the second inequality gives

$$
0 \leq \lambda^{\prime}\left(V\left(c_{\lambda^{\prime}}\right)-V\left(c_{\lambda}\right)\right) \leq E_{0}\left(c_{\lambda}\right)-E_{0}\left(c_{\lambda^{\prime}}\right) .
$$


Now fix some $\lambda_{0}>0$ and use Proposition 7.1 in the Lemmata that follow to obtain estimates uniform in $\lambda \leq \lambda_{0}$, without any reference to the equations. Set

$$
B\left(\lambda_{0}\right)=E_{0}\left(c_{\lambda_{0}}\right)
$$

The following two lemmata follow $[\mathrm{R}]$. The first uses the term in the energy involving the derivative of $H_{\lambda}$ to obtain bounds on $H_{\lambda}$.

Lemma 7.2. For all $r>0$, and for all $\lambda$ in $\left[0, \lambda_{0}\right]$ :

$$
\left|1-\frac{H_{\lambda}(r)}{r}\right| \leq \sqrt{\frac{B\left(\lambda_{0}\right)}{r}},
$$

hence

$$
1-\sqrt{\frac{B\left(\lambda_{0}\right)}{r}} \leq\left|\frac{H_{\lambda}(r)}{r}\right|
$$

Proof. For $0<r<R<\infty$,

$$
\begin{aligned}
\left|\frac{H_{\lambda}(R)}{R}-\frac{H_{\lambda}(r)}{r}\right| & =\left|\int_{r}^{R}\left(\frac{H_{\lambda}(\rho)}{\rho}\right)^{\prime} d \rho\right| \\
& =\left|\int_{r}^{R} \frac{1}{\rho} \rho\left(\frac{H_{\lambda}(\rho)}{\rho}\right)^{\prime} d \rho\right| \\
& \leq\left(\int_{r}^{R} \frac{1}{\rho^{2}} d \rho\right)^{1 / 2}\left(\int_{r}^{R} \rho^{2}\left[\left(\frac{H_{\lambda}(\rho)}{\rho}\right)^{\prime}\right]^{2} d \rho\right)^{1 / 2} \\
& \leq\left(\frac{1}{r}-\frac{1}{R}\right)^{1 / 2}\left(\int_{r}^{R}\left(H_{\lambda}^{\prime}(\rho)-\frac{H_{\lambda}(\rho)}{\rho}\right)^{2} d \rho\right)^{1 / 2}
\end{aligned}
$$

Then for every $\lambda \in\left[0, \lambda_{0}\right]$, Proposition 7.1 gives that $E_{0}\left(A_{\lambda}, \Phi_{\lambda}\right) \leq B\left(\lambda_{0}\right)$. Thus

$$
\int_{r}^{R}\left(H_{\lambda}^{\prime}(\rho)-\frac{H_{\lambda}(\rho)}{\rho}\right)^{2} d \rho \leq B\left(\lambda_{0}\right)
$$

Taking $R \rightarrow \infty$ gives (14). Then (15) follows immediately from (14).

The following uses the term in the energy involving the derivative of $K$ to obtain estimates on $K$. 
Lemma 7.3. There is constant $c_{B\left(\lambda_{0}\right)}>0$ depending only on $B\left(\lambda_{0}\right)$ such that

$$
\left|K_{\lambda}(r)\right| \leq c_{B\left(\lambda_{0}\right)}
$$

for all $r \geq 0$ and for all $\lambda$ in $\left[0, \lambda_{0}\right]$.

Proof. As before, for $0<x<y$

$$
\begin{aligned}
\left|K_{\lambda}(x)^{2}-K_{\lambda}(y)^{2}\right|^{2} & =\left|\int_{x}^{y} 2 K_{\lambda}(r) K_{\lambda}^{\prime}(r) d r\right|^{2} \\
& \leq 4 \int_{x}^{y} K_{\lambda}(r)^{2} d r \int_{x}^{y}\left(K_{\lambda}^{\prime}(r)\right)^{2} d r \\
& \leq 4 B\left(\lambda_{0}\right) \int_{x}^{y} K_{\lambda}(r)^{2} d r
\end{aligned}
$$

To bound the integral in the last term use inequality 15 of Lemma 7.2 to fix $r_{0}>2 B\left(\lambda_{0}\right)$ such that for all $r \geq r_{0}$

$$
0<1 / 2<\left(1-\sqrt{\frac{B\left(\lambda_{0}\right)}{r}}\right)^{2} \leq\left(\frac{H_{\lambda}(r)}{r}\right)^{2} .
$$

Now use the third term in the energy to have

$$
\int_{x}^{y} K_{\lambda}(r)^{2} d r \leq 2 \int_{x}^{y} \frac{K_{\lambda}^{2} H_{\lambda}^{2}}{r^{2}} d r \leq 2 B\left(\lambda_{0}\right),
$$

for $r_{0} \leq x \leq y$. Therefore for such $x$ and $y$

$$
\left|K_{\lambda}(x)^{2}-K_{\lambda}(y)^{2}\right|^{2} \leq 8 B\left(\lambda_{0}\right)^{2}
$$

and, using the boundary condition for $K$ as $x \rightarrow \infty$,

$$
\left|K_{\lambda}(y)\right|^{4} \leq 8 B\left(\lambda_{0}\right)^{2}
$$

which proves the statement for $y \geq r_{0}$. For when $0 \leq y \leq r_{0}$ note that

$$
\begin{aligned}
\left|K_{\lambda}(y)\right| & \leq\left|K_{\lambda}\left(r_{0}\right)\right|+\left|\int_{y}^{r_{0}} K_{\lambda}^{\prime}(r) d r\right| \\
& \leq\left|K_{\lambda}\left(r_{0}\right)\right|+\left(r_{0}-y\right)^{1 / 2} B\left(\lambda_{0}\right)^{1 / 2} \\
& \leq\left|K_{\lambda}\left(r_{0}\right)\right|+r_{0}^{1 / 2} B\left(\lambda_{0}\right)^{1 / 2},
\end{aligned}
$$

with $\left|K_{\lambda}\left(r_{0}\right)\right|$ itself bounded by (16). 
Proposition 7.4. For any $a$ and $b$ with $0<a<b<\infty$ the following hold as $\lambda \rightarrow 0$ :

1. $K_{\lambda}$ converges to $K_{0}$ weakly in $L_{1}^{2}[0, b]$, therefore strongly in $C^{0}[0, b]$, therefore pointwise.

2. $H_{\lambda}$ converges to $H_{0}$ weakly in $L_{1}^{2}[a, b]$, therefore strongly in $C^{0}[a, b]$, therefore pointwise.

Proof. To show (1), define an equivalent norm on $L_{1}^{2}[0, b]$ by

$$
\|f\|_{1}=\left(\int_{0}^{b}\left(f^{\prime}\right)^{2} d r+|f(0)|^{2}\right)^{1 / 2}
$$

and note that Lemma 7.3 and the first term in the energy give that for any $b>0$ and all $\lambda \leq \lambda_{0}$

$$
\left\|K_{\lambda}\right\|_{1} \leq\left(B\left(\lambda_{0}\right)+C_{B\left(\lambda_{0}\right)}^{2}\right)^{1 / 2} .
$$

For (2), define an equivalent norm on $L_{1}^{2}[a, b]$ by

$$
\|f\|_{2}=\left(\int_{a}^{b} r^{2}\left(f^{\prime}\right)^{2} d r+|f(a)|^{2}\right)^{1 / 2}
$$

and note that Lemma 7.2 and the second term in the energy give that for any $a>0$ and for all $\lambda \leq \lambda_{0}$

$$
\left\|\frac{H_{\lambda}(r)}{r}-1\right\|_{2} \leq \sqrt{B\left(\lambda_{0}\right)+\frac{B\left(\lambda_{0}\right)}{a}} .
$$

Therefore both $\left\|K_{\lambda}\right\|_{1}$ and $\left\|1-\frac{H_{\lambda}(r)}{r}\right\|_{2}$ are bounded for $\lambda \leq \lambda_{0}$, therefore any sequence has weakly convergent subsequence as $\lambda \rightarrow 0$. To see that these limits are $\left(K_{0}, H_{0}\right)$ for all sequences, note that since $\left(K_{\lambda}, H_{\lambda}\right)$ solve (YMH1-2) for $\lambda$, the weak limit as $\lambda \rightarrow 0$ solves (YMH1-2) for $\lambda=0$. Then use the uniqueness of the spherically symmetric solution for $\lambda=0$, see $[\mathrm{M}]$.

By the standard embedding theorems these limits are strong in $C^{0}$ and therefore pointwise.

\section{Estimates Uniform in $\lambda$ FOR $K_{\lambda}$}

First the comparison method is used to obtain exponential decay for $K_{\lambda}$, uniform in $\lambda$ and on domains of the form $\left[r_{0}, \infty\right)$, for $r_{0}$ independent of $\lambda$.

As before, $B\left(\lambda_{0}\right)=E_{0}\left(c_{\lambda_{0}}\right)$ for some fixed $\lambda_{0}$. 
Proposition 8.1. There exist $\alpha>0$ and $r_{0}>0$ such that

$$
\left|K_{\lambda}(r)\right| \leq \alpha e^{-r / 2}
$$

for all $r \geq r_{0}$ and for all $\lambda$ in $\left[0, \lambda_{0}\right]$.

Proof. By (15) of Lemma 7.2, for all $r \geq \max \left\{2,2 B\left(\lambda_{0}\right)\right\}$

$$
1<H_{\lambda}^{2}(r)
$$

hence the coefficient of $K_{\lambda}$ in (YMH-1) satisfies

$$
0<\frac{H_{\lambda}^{2}(r)-1+K_{\lambda}^{2}(r)}{r^{2}}
$$

Also note that the limit

$$
\lim _{r \rightarrow \infty} \frac{H_{\lambda}^{2}(r)-1+K_{\lambda}^{2}(r)}{r^{2}}
$$

exists (and it is equal to 1 ) by Lemma 7.2 .

Now let

$$
s(r)=\alpha e^{-r / 2}
$$

be the comparison function, for $\alpha$ to be determined. The claim here is that there exists $r_{0} \geq \max \left\{2,2 B\left(\lambda_{0}\right)\right\}$ such that for all $r \geq r_{0}$ and for all $\lambda \in\left[0, \lambda_{0}\right]$

$$
\left(s(r) \pm K_{\lambda}(r)\right)^{\prime \prime} \leq \frac{H_{\lambda}^{2}(r)-1+K_{\lambda}^{2}(r)}{r^{2}}\left(s(r) \pm K_{\lambda}(r)\right) .
$$

When this is the case, choose $\alpha>0$ depending on $c_{B}$ of Lemma 7.3 so that

$$
0<s\left(r_{0}\right) \pm K_{\lambda}\left(r_{0}\right)
$$

Then conditions (17),(18), (19) and (20) allow the maximum principle, [JT], to be applied and yield

$$
\left|K_{\lambda}(r)\right| \leq \alpha e^{-r / 2}
$$

for $r \geq r_{0}$ and $\lambda \leq \lambda_{0}$.

To check that (19) indeed holds, first note that by (YMH 1-2) (19) is equivalent to

$$
s(r)^{\prime \prime} \leq \frac{H_{\lambda}^{2}(r)-1+K_{\lambda}^{2}(r)}{r^{2}} s(r)
$$


which in turn is equivalent to

$$
\frac{\alpha e^{-r / 2}}{4} \leq \frac{H_{\lambda}^{2}(r)-1+K_{\lambda}^{2}(r)}{r^{2}} \alpha e^{-r / 2}
$$

i.e.

$$
\frac{1}{4} \leq \frac{H_{\lambda}^{2}(r)-1+K_{\lambda}^{2}(r)}{r^{2}}
$$

For the last inequality to hold it is enough that:

$$
\frac{1}{4} \leq \frac{H_{\lambda}^{2}(r)}{r^{2}}-\frac{1}{r^{2}}
$$

By (15) of Lemma 7.2 it is enough to take $r_{0}>2+\sqrt{B\left(\lambda_{0}\right)^{2}+1}$.

Now it is easy to obtain estimates for $K_{\lambda}^{\prime}$ and $K_{\lambda}^{\prime \prime}$.

Lemma 8.2. The estimate of the previous proposition is also valid for $K_{\lambda}^{\prime}$ and $K_{\lambda}^{\prime \prime}$ for possibly different values of $\alpha$.

Proof. For all $r>1$ and for all $\lambda \leq \lambda_{0}$

$$
\left|\frac{H_{\lambda}^{2}}{r^{2}}-\frac{1}{r^{2}}+\frac{K_{\lambda}^{2}}{r^{2}}\right| \leq\left(\sqrt{B\left(\lambda_{0}\right)}+1\right)^{2}+1+c_{B\left(\lambda_{0}\right)}^{2}
$$

by (15) of Lemma 7.2 and Lemma 7.3. Then, for a new $\alpha$ (YMH-1) gives

$$
\left|K_{\lambda}^{\prime \prime}(r)\right| \leq \alpha e^{-r / 2}
$$

for $r \geq r_{0}$ where $r_{0}$ is specified in Proposition 8.1.

This, and the finite energy condition give that $K_{\lambda}^{\prime}$ is in $L_{1}^{2}\left[r_{0}, \infty\right]$. ¿From this it is standard to conclude that

$$
\lim _{r \rightarrow \infty} K_{\lambda}^{\prime}(r)=0
$$

see for example [JT], page 86. Using this,

$$
\begin{aligned}
\left|K_{\lambda}^{\prime}(r)\right| & =\left|\int_{r}^{\infty} K_{\lambda}^{\prime \prime}(\rho) d \rho\right| \\
& \leq \int_{r}^{\infty} \alpha e^{-\rho / 2} d \rho \\
& =2 \alpha e^{-r / 2}
\end{aligned}
$$

for all $r \geq r_{0}$ and for all $\lambda$ in $\left[0, \lambda_{0}\right]$. 


\section{Estimates FOR THE DECAY OF $1-\frac{H_{\lambda}(r)}{r}$}

To obtain estimates for $H_{\lambda}, H_{\lambda}^{\prime}$ and $H_{\lambda}^{\prime \prime}$, by (YMH-2) sharper estimates on $\left|1-\frac{H_{\lambda}(r)}{r}\right|$ are in order. The following two propositions improve on Lemma 7.2.

First estimate exponential decay which, unlike that of $K_{\lambda}$, depends on $\lambda$ and, for the first time, imposes restrictions on $\lambda_{0}$.

Proposition 9.1. There exist $\alpha>0, r_{0}>0$ such that for all $\lambda<\frac{1}{8}$ and for all $r \geq r_{0}$

$$
\left|1-\frac{H_{\lambda}(r)}{r}\right| \leq \alpha e^{-\sqrt{\lambda} r}
$$

Proof. Let $u_{\lambda}(r)=1-\frac{H_{\lambda}(r)}{r}$. Differentiate twice to obtain

$$
u_{\lambda}^{\prime \prime}+\frac{2}{r} u_{\lambda}^{\prime}=-\frac{H_{\lambda}^{\prime \prime}}{r}
$$

Replacing $H_{\lambda}^{\prime \prime}$ from (YMH-2) yields

$$
u_{\lambda}^{\prime \prime}+\frac{2}{r} u_{\lambda}^{\prime}-\frac{4 \lambda H_{\lambda}}{r}\left(1+\frac{H_{\lambda}}{r}\right) u_{\lambda}=-\frac{2 K_{\lambda}^{2} H_{\lambda}}{r^{3}} .
$$

Now let

$$
s(r)=\alpha e^{-\sqrt{\lambda} r}-e^{-r}
$$

to be the test function. The aim is to show that there exist $\alpha>0$ and $r_{0}>0$ such that

$$
\left|u_{\lambda}(r)\right| \leq s(r)
$$

for all $r \geq r_{0}$ and for all $\lambda$ in an appropriate range. The term $-e^{-r}$ in the formula of $s$ seems to be necessary for the maximum principle and it is imposed by the form of (21). By 
Proposition 8.1 and Lemma 7.2, for $\lambda$ smaller than some $\lambda_{0}$ :

$$
\begin{aligned}
& \left(s \pm u_{\lambda}\right)^{\prime \prime}+\frac{2}{r}(s \pm u)^{\prime}-\frac{4 \lambda H_{\lambda}}{r}\left(1+\frac{H_{\lambda}}{r}\right)\left(s \pm u_{\lambda}\right) \\
= & \mp \frac{2 K_{\lambda}^{2} H_{\lambda}}{r^{3}}+\alpha \lambda e^{-\sqrt{\lambda} r}-e^{-r}+\frac{2}{r}\left(-\alpha \sqrt{\lambda} e^{-\sqrt{\lambda} r}+e^{-r}\right) \\
& -\frac{4 \lambda H_{\lambda}}{r}\left(1+\frac{H_{\lambda}}{r}\right)\left(\alpha e^{-\sqrt{\lambda} r}-e^{-r}\right) \\
\leq & \frac{2}{r^{2}} e^{-r}-e^{-r}+\frac{2}{r} e^{-r}+\frac{4 \lambda H_{\lambda}}{r}\left(1+\frac{H_{\lambda}}{r}\right) e^{-r} \\
& +\left(\lambda-\frac{2 \sqrt{\lambda}}{r}-\frac{4 \lambda H_{\lambda}}{r}\left(1+\frac{H_{\lambda}}{r}\right)\right) \alpha e^{-\sqrt{\lambda} r} \\
\leq & \left(\frac{2}{r^{2}}-1+\frac{2}{r}+8 \lambda\right) e^{-r}+\left(\lambda-\frac{2 \sqrt{\lambda}}{r}-4 \lambda\left(1-\frac{\sqrt{B\left(\lambda_{0}\right)}}{\sqrt{r}}\right)\left(1+1-\frac{\sqrt{B\left(\lambda_{0}\right)}}{\sqrt{r}}\right)\right) \alpha e^{-\sqrt{\lambda} r} \\
\leq & \left(\frac{2}{r^{2}}-1+\frac{2}{r}+8 \lambda\right) e^{-r}+\left(\lambda\left(-7+\frac{4 \sqrt{B\left(\lambda_{0}\right)}}{\sqrt{r}}+8 \sqrt{\frac{B\left(\lambda_{0}\right)}{r}}\right)-\frac{2 \sqrt{\lambda}}{r}\right) \alpha e^{-\sqrt{\lambda} r} .
\end{aligned}
$$

Therefore set $\lambda_{0}=\frac{1}{8}$ and choose $r_{0}>0$ such that

$$
\left(s \pm u_{\lambda}\right)^{\prime \prime}+\frac{2}{r}(s \pm u)^{\prime}-\frac{4 \lambda H_{\lambda}}{r}\left(1+\frac{H_{\lambda}}{r}\right)\left(s \pm u_{\lambda}\right)<0,
$$

for all $r \geq r_{0}$ and $\lambda$ in $[0,1 / 8)$.

Now use for all $\lambda<1 / 8$ the bound

$$
\left|u_{\lambda}\left(r_{0}\right)\right| \leq 1+\sqrt{\frac{B(1 / 8)}{r_{0}}}
$$

of Lemma 7.2 to choose $\alpha>0$ such that

$$
0<s\left(r_{0}\right) \pm u_{\lambda}\left(r_{0}\right)
$$

To obtain a uniform in $\lambda$ estimate on the decay of $\left|1-\frac{H_{\lambda}(r)}{r}\right|$, one has to make do with power low decay: 
Proposition 9.2. There exist $\alpha>0$ and $r_{0}>0$ such that for all $\lambda$ in $[0,1 / 8)$ and for all $r \geq r_{0}$

$$
\left|1-\frac{H_{\lambda}(r)}{r}\right| \leq \frac{\alpha}{r} .
$$

Proof. Repeat the proof of 9.1 for the same $u_{\lambda}$ but now change the comparison function to

$$
s(r)=\frac{\alpha}{r}-e^{-r}
$$

where $\alpha$ is specified below.

Again, Lemma 7.2 and Proposition 8.1 give for any $\lambda$ smaller than some $\lambda_{0}$

$$
\begin{aligned}
& \left(s \pm u_{\lambda}\right)^{\prime \prime}+\frac{2}{r}(s \pm u)^{\prime}-\frac{4 \lambda H_{\lambda}}{r}\left(1+\frac{H_{\lambda}}{r}\right)\left(s \pm u_{\lambda}\right) \\
= & \mp \frac{2 K_{\lambda}^{2} H_{\lambda}}{r^{3}}-e^{-r}+\frac{2 e^{-r}}{r}-\frac{4 \lambda H_{\lambda}}{r}\left(1+\frac{H_{\lambda}}{r}\right) \frac{\alpha}{r}+\frac{4 \lambda H_{\lambda}}{r} e^{-r} \\
\leq & \frac{2 e^{-r}}{r^{2}}-e^{-r}+\frac{2 e^{-r}}{r}-4 \lambda\left(1+1-\frac{B\left(\lambda_{0}\right)}{\sqrt{r}}\right) \frac{\alpha}{r}+8 \lambda e^{-r} \\
= & \left(\frac{2}{r^{2}}-1+\frac{2}{r}+8 \lambda\right) e^{-r}-4 \lambda\left(2-\frac{B\left(\lambda_{0}\right)}{\sqrt{r}}\right) \frac{\alpha}{r} .
\end{aligned}
$$

Now set $\lambda_{0}=\frac{1}{8}$ and choose $r_{0}>0$ such that

$$
\left(s \pm u_{\lambda}\right)^{\prime \prime}+\frac{2}{r}(s \pm u)^{\prime}-\frac{4 \lambda H_{\lambda}}{r}\left(1+\frac{H_{\lambda}}{r}\right)\left(s \pm u_{\lambda}\right)<0,
$$

for all $r \geq r_{0}$, and $\lambda$ in $[0,1 / 8)$. Finally, choose $\alpha>0$ such that

$$
0<s\left(r_{0}\right) \pm u_{\lambda}\left(r_{0}\right)
$$

$\lambda$ in $[0,1 / 8)$

Here are two immediate applications of the last proposition.

Corollary 9.3. There exists $M>0$ such that for $\lambda$ in $[0,1 / 8)$ the following holds for $r \geq 0$

$$
\left|H_{\lambda}(r)-H_{0}(r)\right| \leq M
$$

Proof. By Proposition 9.2 there is $r_{0}$ satisfying

$$
\left|r-H_{\lambda}(r)\right| \leq \alpha
$$


for $r \geq r_{0}$. It is also immediate that

$$
\left|H_{0}(r)-r\right| \leq 1
$$

for all $r \geq 0$. Thus the required estimate follows for $r \geq r_{0}$. Also, since $\left|H_{\lambda}(r)\right| \leq r$ for all $\lambda \geq 0$ and $r \geq 0$, see Theorem $I V .10 .1$ of $[\mathrm{JT}]$, the result follows.

\section{Corollary 9.4.}

$$
\lim _{r \rightarrow \infty} H_{\lambda}^{\prime}(r)=1
$$

for all $\lambda<1 / 8$.

Proof. Recall that on one dimension functions in $L_{1}^{2}$ are zero at infinity, see Proposition 7.5 of [JT]. To use this, estimate that for the $L^{2}$ norm on $\left[r_{0}, \infty\right)$, for $r_{0}$ as in Proposition 9.2

$$
\left\|H_{\lambda}^{\prime}-1\right\|_{2} \leq\left\|H_{\lambda}^{\prime}-\frac{H_{\lambda}}{r}\right\|_{2}+\left\|\frac{H_{\lambda}}{r}-1\right\|_{2}<\infty
$$

since the first term on the right hand side is part of the energy and the second term is finite by 9.2 for $\lambda<1 / 8$.

For the derivative of $H_{\lambda}^{\prime}-1$ estimate using (YMH-2)

$$
\left\|H_{\lambda}^{\prime \prime}\right\|_{2} \leq 2\left\|\frac{K_{\lambda}^{2}}{r^{2}} H_{\lambda}\right\|_{2}+4 \lambda\left\|H_{\lambda}\left(1-\frac{H_{\lambda}}{r}\right)\left(1+\frac{H_{\lambda}}{r}\right)\right\|_{2} .
$$

For the first term of the right hand side recall Proposition 8.1 and that $\left|H_{\lambda}(r)\right|<r$, see Theorem 10.1 of [JT]. For the second term use Proposition 9.1 and that

$$
\left\|r e^{-\sqrt{\lambda} r}\right\|_{L^{2}[0, \infty]}=\frac{1}{2 \lambda^{3 / 4}}
$$

Now estimate the behavior of $H_{\lambda}^{\prime}$ at infinity.

Proposition 9.5. For every $p \in(3 / 2,2)$ there exist $r_{0}, \alpha>0$ such that

$$
\left|\left(\frac{H_{\lambda}}{r}\right)^{\prime}\right| \leq \frac{\alpha}{r^{p}}
$$

for all $r \geq r_{0}$ and for all $\lambda$ in $[0,1 / 8)$. 
Proof. Set

$$
v_{\lambda}(r)=\left(\frac{H_{\lambda}}{r}\right)^{\prime} .
$$

Differentiate (21) to obtain

$$
v_{\lambda}^{\prime \prime}+\frac{2}{r} v_{\lambda}^{\prime}-\left(\frac{2}{r^{2}}+4 \lambda\left(2-3\left(1-\frac{H_{\lambda}^{2}}{r^{2}}\right)\right)+\frac{2 K_{\lambda}^{2}}{r^{2}}\right) v_{\lambda}=-2\left(\frac{K_{\lambda}^{2}}{r^{2}}\right)^{\prime} \frac{H_{\lambda}}{r}
$$

For $p \in(3 / 2,2)$ set

$$
s(r)=\frac{\alpha}{r^{p}}-e^{-r},
$$

for $\alpha$ to be determined again.

Then

$$
s^{\prime \prime}+\frac{2}{r} s^{\prime}=\alpha \frac{p(p-1)}{r^{p+2}}-\left(1-\frac{2}{r}\right) e^{-r} .
$$

By Lemma 8.2 and Proposition 9.2 there is $r_{0}$ and $A$ such that for all $r>r_{0}$ and $\lambda<1 / 8$

$$
\begin{aligned}
&\left(s \pm v_{\lambda}\right)^{\prime \prime}+\frac{2}{r}\left(s \pm v_{\lambda}\right)^{\prime}-\left(\frac{2}{r^{2}}+4 \lambda\left(2-3\left(1-\frac{H_{\lambda}^{2}}{r^{2}}\right)\right)+\frac{2 K_{\lambda}^{2}}{r^{2}}\right)\left(s \pm v_{\lambda}\right) \\
&= \mp 2\left(\frac{K_{\lambda}^{2}}{r^{2}}\right)^{\prime} \frac{H_{\lambda}}{r}+\alpha \frac{p(p-1)}{r^{p+2}}-\left(1-\frac{2}{r}\right) e^{-r}-\frac{2 \alpha}{r^{p+2}}+\frac{12 \lambda \alpha}{r^{p}}\left(1-\frac{H_{\lambda}^{2}}{r^{2}}\right)-\frac{8 \lambda \alpha}{r^{p}}-\frac{2 K_{\lambda}^{2} \alpha}{r^{p+2}} \\
&+\left(\frac{2}{r^{2}}+12 \lambda\left(\frac{H_{\lambda}^{2}}{r^{2}}-1\right)+8 \lambda+\frac{2 K_{\lambda}^{2}}{r^{2}}\right) e^{-r} \\
& \leq \frac{4\left|K_{\lambda} K_{\lambda}^{\prime}\right|}{r^{2}}+\frac{4 K_{\lambda}^{2}}{r^{3}}-\frac{2-p(p-1)}{r^{p+2}} \alpha-\left(1-\frac{2}{r}\right) e^{-r}+\frac{24 \lambda A}{r^{p+1}} \alpha-\frac{8 \lambda}{r^{p}} \alpha-\frac{2 K_{\lambda}^{2}}{r^{p+2}} \alpha \\
&+\left(\frac{2}{r^{2}}+8 \lambda+\frac{2 e^{-r}}{r^{2}}\right) e^{-r} \\
& \leq \frac{4 A^{2} e^{-r}}{r^{2}}+\frac{4 A^{2} e^{-r}}{r^{3}}-\left(1-\frac{2}{r}\right) e^{-r}-8 \lambda \alpha\left(1-\frac{3 A}{r}\right) \frac{1}{r^{p}}-\frac{2-p(p-1)}{r^{p+2}} \alpha \\
&+\left(\frac{2}{r^{2}}+8 \lambda+\frac{2 e^{-r}}{r^{2}}\right) e^{-r} .
\end{aligned}
$$

Now, for $\lambda$ still in $[0,1 / 8)$, choose a different $r_{0}$ so that for $r>r_{0}$ this last quantity becomes negative:

$$
\begin{gathered}
\frac{4 A^{2}}{r^{2}}+\frac{4 A^{2}}{r^{3}}-\left(1-\frac{2}{r}\right)<0, \\
-8 \lambda \alpha\left(1-\frac{3 A}{r}\right) \frac{1}{r^{p}}<0, \\
-\frac{2-p(p-1)}{r^{p+2}}+\left(\frac{2}{r^{2}}+8 \lambda+\frac{2 e^{-r}}{r^{2}}\right) e^{-r}<0,
\end{gathered}
$$


where to make the last inequality valid $\alpha$ will have to be greater than 1 . Since the $L^{\infty}(0, \infty)$ norm of $v_{\lambda}$ is uniformly bounded for $\lambda$ in any bounded interval by Proposition 7.1 and Theorem IV.11.1 of $[\mathrm{JT}]$, such that $s\left(r_{0}\right) \pm v_{\lambda}\left(r_{0}\right)>0$ for all $\lambda \in[0,1 / 8)$.

\section{Estimates Uniform IN $\lambda$ FOR $H_{\lambda}$ AND $K_{\lambda}$ AT 0}

The next proposition describes the behavior of $H_{\lambda}$ and $K_{\lambda}$ near zero. The technique here is an adaptation to uniform estimates of the technique in $[R]$ for individual ones.

Proposition 10.1. There exists $M>0$ and $\lambda_{0}>0$ such that

$$
\left|\frac{H_{\lambda}(r)}{r^{2}}\right| \leq M
$$

and

$$
\left|\frac{1-K_{\lambda}}{r^{2}}\right| \leq M
$$

for all $r$ in $[0,1]$ and $\lambda$ in $\left[0, \lambda_{0}\right]$.

Proof. The proof consists of four steps:

Step 1: For every $\lambda_{0}>0$ the set $\left(\frac{H_{\lambda}(r)}{r^{3 / 2}}\right)_{\lambda \in\left[0, \lambda_{0}\right]}$ is bounded in $C[0,1]$ :

by (YMH 2)

$$
r H_{\lambda}^{\prime \prime}-\frac{2 H_{\lambda}}{r}=\frac{2\left(K_{\lambda}^{2}-1\right) H_{\lambda}}{r}+\frac{4 \lambda H_{\lambda}^{3}}{r}-4 \lambda r H_{\lambda}
$$

and the left hand side now equals

$$
\frac{1}{r}\left(r^{3}\left(\frac{H_{\lambda}}{r}\right)^{\prime}-r^{2} \frac{H_{\lambda}}{r}\right)^{\prime}
$$

Rewriting (24) as

$$
\left(r^{3}\left(\frac{H_{\lambda}}{r}\right)^{\prime}-r^{2} \frac{H_{\lambda}}{r}\right)^{\prime}=r^{3}\left(2 \frac{K_{\lambda}^{2}-1}{r}-4 \lambda r\left(1-\frac{H_{\lambda}^{2}}{r^{2}}\right)\right) \frac{H_{\lambda}}{r^{2}},
$$

integrating both sides from $y$ to $x$ for some $0<y<x$ and then to taking $y \rightarrow 0$ while noticing that the extra $y$-terms go to zero gives

$$
x^{3}\left(\frac{H_{\lambda}}{x}\right)^{\prime}-x^{2} \frac{H_{\lambda}}{x}=\int_{0}^{x} r^{3}\left(2 \frac{K_{\lambda}^{2}-1}{r}-4 \lambda r\left(1-\frac{H_{\lambda}^{2}}{r^{2}}\right)\right) \frac{H_{\lambda}}{r^{2}} d r .
$$


Noting that the left hand side equals $x^{4}\left(\frac{H_{\lambda}}{x^{2}}\right)^{\prime}$, set

$$
h_{\lambda}(r)=2 \frac{K_{\lambda}^{2}-1}{r}-4 \lambda r\left(1-\frac{H_{\lambda}^{2}}{r^{2}}\right)
$$

Note that the $L^{2}[0,1]$ norms of the functions $\left(h_{\lambda}\right)_{\lambda \in\left[0, \lambda_{0}\right]}$ are uniformly bounded by the monotonicity of the energies and the estimate $\left|H_{\lambda}(r)\right| \leq r$ for all $r \geq 0$ and for all $\lambda \geq 0$.

In addition,

$$
x^{4}\left(\frac{H_{\lambda}}{x^{2}}\right)^{\prime}=\int_{0}^{x} r^{3} h_{\lambda} \frac{H_{\lambda}}{r^{2}} d r .
$$

and

$$
\left|r^{2} \frac{H_{\lambda}(r)}{r^{2}}\right| \leq 1
$$

for all $r$ in $[0,1]$ and $\lambda \geq 0$. This will be the first step in a bootstrapping which uses the following Lemma. The Lemma is stated in a general form for it will be applied repeatedly during the course of this proof. The proof of the Lemma itself is postponed for the moment.

Lemma 10.2. Let $\alpha, \beta, \gamma$, and $k$ be positive numbers such that $0<\gamma-\alpha+3 / 2<k$ and $\alpha \leq \beta+1$. Let $f_{\lambda}, g_{\lambda}$, and $h_{\lambda}$ be functions defined on $[0,1]$ where $\lambda$ runs in some parameter set $\Lambda$. Assume that

1. the functions $f_{\lambda}$ are differentiable on $[0,1]$

2. the set $\left(f_{\lambda}(1)\right)_{\lambda \in \Lambda}$ is bounded

3. the set $\left(g_{\lambda}\right)_{\lambda \in \Lambda}$ is bounded in $C[0,1]$

4. the $L^{2}[0,1]$ norm of the functions $\left(h_{\lambda}\right)_{\lambda \in \Lambda}$ is uniformly bounded

5. the functions $f_{\lambda}, g_{\lambda}$, and $h_{\lambda}$ satisfy

$$
x^{\alpha}\left|f_{\lambda}^{\prime}(x)\right| \leq \int_{0}^{x} r^{\beta}\left|g_{\lambda}(r)\right| d r+\int_{0}^{x} r^{\gamma}\left|f_{\lambda}(r) h_{\lambda}(r)\right| d r
$$

and

6. the set of functions $\left(r^{k} f_{\lambda}(r)\right)_{\lambda \in \Lambda}$ is bounded in $C[0,1]$.

Then the set of functions $\left(r^{k-(\gamma-\alpha+3 / 2)} f_{\lambda}(r)\right)_{\lambda \in \Lambda}$ is also bounded in $C[0,1]$. 
Since $H_{\lambda}(1)<1$ for all $\lambda$, use equations (26) and (27) and apply the above lemma for

$$
\Lambda=\left[0, \lambda_{0}\right], \quad \alpha=4, \quad f_{\lambda}(r)=\frac{H_{\lambda}(r)}{r^{2}}, \quad g_{\lambda}(r)=0, \quad h_{\lambda}(r) \text { as in }(25), \quad \gamma=3, \quad k=2,
$$

to have that the set $\left(r^{3 / 2} f_{\lambda}(r)\right)_{\lambda \in\left[0, \lambda_{0}\right]}$ is bounded in $C[0,1]$.

Repeating twice gives that the set $\left(\frac{H_{\lambda}(r)}{r^{3 / 2}}\right)_{\lambda \in\left[0, \lambda_{0}\right]}$ is bounded in $C[0,1]$.

To bridge the gap between Step 1 and the statement of the Proposition, a similar bootstrapping on $K_{\lambda}$ is in order.

Step 2: There exists $\lambda_{0}>0$ such that the set $\left(\frac{1-K_{\lambda}(r)}{r^{3 / 2}}\right)_{\lambda \in\left[0, \lambda_{0}\right]}$ is bounded in $C[0,1]$.

By (YMH-1) we obtain:

$$
-r^{2} K_{\lambda}^{\prime \prime}-2\left(1-K_{\lambda}\right)=-r^{3} \frac{H_{\lambda}^{2}}{r^{3}} K_{\lambda}+r^{3}\left(\frac{K_{\lambda}^{2}-1}{r}+\frac{K_{\lambda}-1}{r}\right) \frac{1-K_{\lambda}}{r^{2}} .
$$

Noting that the left hand side of this equation equals

$$
\left(r^{3}\left(\frac{1-K_{\lambda}}{r}\right)^{\prime}-r^{2}\left(\frac{1-K_{\lambda}}{r}\right)\right)^{\prime}
$$

set

$$
h_{\lambda}(r)=\frac{K_{\lambda}^{2}-1}{r}+\frac{K_{\lambda}-1}{r}, \quad g_{\lambda}(r)=-\frac{H_{\lambda}^{2}}{r^{3}} K_{\lambda}
$$

to obtain

$$
\left(r^{3}\left(\frac{1-K_{\lambda}}{r}\right)^{\prime}-r^{2}\left(\frac{1-K_{\lambda}}{r}\right)\right)^{\prime}=r^{3} g_{\lambda}+r^{3} h_{\lambda} \frac{1-K_{\lambda}}{r^{2}} .
$$

By the convergence of $K_{\lambda}$ to $K_{0}$ in $C[0,1]$ there exists $\lambda_{0}>0$ such that $K_{\lambda}(r)>0$ for all $\lambda$ in $\left[0, \lambda_{0}\right]$ and for all $r$ in $[0,1]$. (This is the second time that $\lambda_{0}$ need to be restricted, and for the first time in an unknown range.) Thus the second term of $h_{\lambda}$ is dominated by the first one. Therefore by Proposition 7.1 we obtain that the $L^{2}[0,1]$ norms of the functions $h_{\lambda}$ are uniformly bounded. Now integrate both sides from zero to $x$ to obtain:

$$
x^{3}\left(\frac{1-K_{\lambda}(x)}{x}\right)^{\prime}-x^{2} \frac{1-K_{\lambda}(x)}{x}=\int_{0}^{x} r^{3} g_{\lambda} d r+\int_{0}^{x} r^{3} h_{\lambda} \frac{1-K_{\lambda}}{r^{2}} d r
$$

and note that the left hand side equals

$$
x^{4}\left(\frac{1-K_{\lambda}(x)}{x^{2}}\right)^{\prime} .
$$


Thus

$$
x^{4}\left(\frac{1-K_{\lambda}(x)}{x^{2}}\right)^{\prime}=\int_{0}^{x} r^{3} g_{\lambda} d r+\int_{0}^{x} r^{3} h_{\lambda} \frac{1-K_{\lambda}}{r^{2}}, d r
$$

and by Lemma 7.3

$$
\left|r^{2} \frac{1-K_{\lambda}(r)}{r^{2}}\right| \leq 1+C_{B\left(\lambda_{0}\right)}
$$

for all $r$ and $\lambda \in\left[0, \lambda_{0}\right]$.

(30) and (31) become the first step in the bootstrapping when Lemma 10.2 is applied for $g$ and $h$ as in (29) gives that the set $\left(r^{3 / 2} \frac{1-K_{\lambda}(r)}{r^{2}}\right)_{\lambda \in\left[0, \lambda_{2}\right]}$ is bounded in $C[0,1]$.

Apply Lemma 10.2 twice more to obtain that the set

$$
\left(r^{1 / 2} \frac{1-K_{\lambda}(r)}{r^{2}}\right)_{\lambda \in\left[0, \lambda_{0}\right]}
$$

is bounded in $C[0,1]$.

Step 3: Now the estimate on $H_{\lambda}$ can be proved. For this, write (26) as follows:

$$
x^{4}\left(\frac{H_{\lambda}}{x^{2}}\right)^{\prime}=\int_{0}^{x} r^{7 / 2}\left(2 \frac{K_{\lambda}^{2}-1}{r^{3 / 2}}-4 \lambda \sqrt{r}\left(1-\frac{H_{\lambda}^{2}}{r^{2}}\right)\right) \frac{H_{\lambda}}{r^{2}} d r .
$$

Now Lemma 10.2 cannot be applied starting with (32), for $\gamma=7 / 2$, and $k=1 / 2$ because $k<\gamma-\alpha+3 / 2$. However, it suffices to use part of its proof to get the required estimate. Then for $\lambda \in\left[0, \lambda_{0}\right]$ set

$$
h_{\lambda}(r)=2 \frac{K_{\lambda}^{2}-1}{r^{3 / 2}}-4 \lambda \sqrt{r}\left(1-\frac{H_{\lambda}^{2}}{r^{2}}\right)
$$

and note that by Step 2 the $\left\|h_{\lambda}(r)\right\|_{L^{2}[0,1]}$ is uniformly bounded. Combining this with the assertion of Step 1, there exists $C>0$ such that for all $\lambda$ in $\left[0, \lambda_{0}\right]$ :

$$
\left(\int_{0}^{1} h_{\lambda}^{2}(r) d r\right)^{1 / 2} \leq C
$$

and

$$
\left|\frac{H_{\lambda}}{r^{3 / 2}}\right| \leq C
$$


for $r$ in $[0,1]$. Then (32) gives

$$
\begin{aligned}
x^{4}\left(\frac{H_{\lambda}}{x^{2}}\right)^{\prime} & \leq\left(\int_{0}^{1} h_{\lambda}^{2}(r) d r\right)^{1 / 2}\left(\int_{0}^{x} r^{7}\left(\frac{H_{\lambda}}{r^{2}}\right)^{2} d r\right)^{1 / 2} \\
& \leq C\left(\int_{0}^{x} r^{6}\left(\frac{H_{\lambda}}{r^{3 / 2}}\right)^{2} d r\right)^{1 / 2} \\
& \leq C^{2}\left(\int_{0}^{x} r^{6} d r\right)^{1 / 2}=C^{2} x^{7 / 2}
\end{aligned}
$$

Thus for $\lambda$ in $\left[0, \lambda_{0}\right]$ and $x$ in $[0,1]$

$$
\left(\frac{H_{\lambda}}{x^{2}}\right)^{\prime} \leq \frac{C^{2}}{\sqrt{x}}
$$

For $0<r<1$ integrate the last inequality from $r$ to 1 to obtain:

$$
\left|H_{\lambda}(1)-\frac{H_{\lambda}(r)}{r^{2}}\right| \leq 2 C^{2}|1-\sqrt{r}|
$$

for $r$ in $[0,1]$. Since $\left|H_{\lambda}(1)\right|<1$, the estimate for $H_{\lambda}$ follows.

Step 4: Now the statement for $K_{\lambda}$ can be proved. For this write (30) as follows:

$$
x^{4}\left(\frac{1-K_{\lambda}(x)}{x^{2}}\right)^{\prime}=\int_{0}^{x} r^{4}\left(-\frac{H_{\lambda}^{2}}{r^{4}}\right) K_{\lambda} d r+\int_{0}^{x} r^{7 / 2}\left(\frac{K_{\lambda}^{2}-1}{r^{3 / 2}}+\frac{K_{\lambda}-1}{r^{3 / 2}}\right) \frac{1-K_{\lambda}}{r^{2}} d r .
$$

Set

$$
g_{\lambda}(r)=-\frac{H_{\lambda}^{2}}{r^{4}} K_{\lambda}
$$

and

$$
h_{\lambda}(r)=\frac{K_{\lambda}^{2}-1}{r^{3 / 2}}+\frac{K_{\lambda}-1}{r^{3 / 2}} .
$$

By steps 2 and 3 and the choice of $\lambda_{0}$ there exist $C>0$ such that for all $\lambda$ in $\left[0, \lambda_{0}\right]$ :

$$
\left(\int h_{\lambda}^{2}(r) d r\right)^{1 / 2} \leq C
$$

and

$$
\left|g_{\lambda}(r)\right| \leq C
$$


for $r$ in $[0,1]$. Then (33) gives

$$
\begin{aligned}
x^{4}\left(\frac{1-K_{\lambda}}{x^{2}}\right)^{\prime} & \leq C \int_{0}^{x} r^{4} d r+\left(\int_{0}^{1} h_{\lambda}^{2} d r\right)^{1 / 2}\left(\int_{0}^{x} r^{7}\left(\frac{1-K_{\lambda}}{r^{2}}\right)^{2} d r\right)^{1 / 2} \\
& \leq C x^{5}+C\left(\int_{0}^{x} r^{6}\left(\frac{1-K_{\lambda}}{r^{3 / 2}}\right)^{2} d r\right)^{1 / 2} \\
& \leq C x^{5}+C^{2}\left(\int_{0}^{x} r^{6} d r\right)^{1 / 2} \\
& =C\left(x^{7 / 2}+x^{5}\right) .
\end{aligned}
$$

This gives

$$
\left(\frac{1-K_{\lambda}}{x^{2}}\right)^{\prime} \leq C\left(x+\frac{1}{\sqrt{x}}\right)
$$

which integrated from $r$ to 1 gives that

$$
\left|\frac{1-K_{\lambda}}{r^{2}}-\left(1-K_{\lambda}(1)\right)\right| \leq C\left(\frac{r^{2}}{2}+2 \sqrt{r}\right)+C .
$$

The estimate for $K_{\lambda}$ follows.

\section{Proof of Lemma 10.2}

Choose a constant $B>0$ such that for all $\lambda \in \Lambda$

$$
\left(\int_{0}^{1} h_{\lambda}^{2} d r\right)^{1 / 2} \leq B, \quad\left|g_{\lambda}(r)\right| \leq B
$$

for all $r$ in $[0,1]$. Therefore for every $x$ in $[0,1]$

$$
\int_{0}^{x} r^{\beta}\left|g_{\lambda}(r)\right| d r \leq B \int_{0}^{x} r^{\beta} d r=\frac{B}{\beta+1} x^{\beta+1},
$$

and

$$
\begin{aligned}
\int_{0}^{x} r^{\gamma}\left|f_{\lambda} h_{\lambda}\right| d r & \leq\left(\int_{0}^{1} h_{\lambda}^{2}(r) d r\right)^{1 / 2}\left(\int_{0}^{x} r^{2 \gamma} f_{\lambda}^{2}(r) d r\right)^{1 / 2} \\
& \leq B\left(\int_{0}^{x} r^{2(\gamma-k)}\left(r^{k} f_{\lambda}(r)\right)^{2} d r\right)^{1 / 2} \\
& \leq B^{2}\left(\int_{0}^{x} r^{2(\gamma-k)} d r\right)^{1 / 2}=\frac{B^{2} x^{\gamma-k+1 / 2}}{(2(\gamma-k)+1)^{1 / 2}}
\end{aligned}
$$


Thus by equation (28)

$$
\left|f_{\lambda}^{\prime}(x)\right| \leq \frac{B}{\beta+1} x^{\beta+1-\alpha}+\frac{B^{2}}{(2(\gamma-k)+1)^{1 / 2}} \frac{1}{x^{\alpha-\gamma+k-1 / 2}} .
$$

But $k>\gamma-\alpha+3 / 2$ implies $\alpha-\gamma+k-1 / 2 \neq 1$, thus by integrating (34) from $y$ to 1 (for some $0<y \leq 1)$

$$
\left|f_{\lambda}(1)-f_{\lambda}(y)\right| \leq \frac{2 B}{\beta+1}+\frac{B^{2}}{(2(\gamma-k)+1)^{1 / 2}(-\gamma+\alpha+k-3 / 2)}\left|1-\frac{1}{y^{\alpha-\gamma+k-3 / 2}}\right| .
$$

Now set

$$
C=\frac{B^{2}}{\left((2(\gamma-k)+1)^{1 / 2}(-\gamma+\alpha+k-3 / 2)\right.}
$$

and multiply the last inequality by $y^{\alpha-\gamma+k-3 / 2}$ to have

$$
\left|f_{\lambda}(1) y^{\alpha-\gamma+k-3 / 2}-f_{\lambda}(y) y^{\alpha-\gamma+k-3 / 2}\right| \leq \frac{2 B y^{\alpha-\gamma+k-3 / 2}}{\beta+1}+C\left|y^{\alpha-\gamma+k-3 / 2}-1\right|
$$

Now since $k>\gamma-\alpha+3 / 2$, and $\left(f_{\lambda}(1)\right)_{\lambda \in \Lambda}$ is bounded, the set

$$
\left(f_{\lambda}(y) y^{\alpha-\gamma+k-3 / 2}\right)_{\lambda \in \Lambda}
$$

is bounded in $C[0,1]$.

Proposition 10.3. There exists $M>0$ and $\lambda_{0}>0$ such that

$$
\left|\frac{H_{\lambda}^{\prime}(r)}{r}\right| \leq M
$$

and

$$
\left|\frac{K_{\lambda}^{\prime}(r)}{r}\right| \leq M
$$

for all $r$ in $[0,1]$ and $\lambda$ in $\left[0, \lambda_{0}\right]$.

Proof. Computation of the left hand side of (26) gives:

$$
\left|x^{2} H_{\lambda}^{\prime}-2 x H_{\lambda}\right| \leq \int_{0}^{x} r^{3}\left|h_{\lambda} \frac{H_{\lambda}}{r^{2}}\right| d r
$$

for $x$ in $[0,1]$. By Proposition 10.1 there exists $C>0$ such that

$$
\left|h_{\lambda} \frac{H_{\lambda}}{r^{2}}\right| \leq C
$$


for $r$ in $[0,1]$ and $\lambda$ in $\left[0, \lambda_{0}\right]$. Therefore the last inequality gives:

$$
\left|x^{2} H_{\lambda}^{\prime}-2 x H_{\lambda}\right| \leq C x^{4}
$$

Dividing by $x^{3}$ yields:

$$
\left|\frac{H_{\lambda}^{\prime}}{x}\right| \leq C x+\left|\frac{H_{\lambda}}{x^{2}}\right|
$$

which gives the result for $H_{\lambda}^{\prime}$ by virtue of Proposition 10.1.

The estimate for $K_{\lambda}^{\prime}$ follows along similar lines: Computation of the left hand side of (30) gives:

$$
\left|-x^{2} K_{\lambda}^{\prime}-2 x\left(1-K_{\lambda}\right)\right| \leq \int_{0}^{x} r^{3}\left|g_{\lambda}\right| d r+\int_{0}^{x} r^{3}\left|h_{\lambda} \frac{1-K_{\lambda}}{r^{2}}\right| d r
$$

for $x$ in $[0,1]$. By Propositions 10.1 and 7.3 there exists $C>0$ such that

$$
\left|g_{\lambda}(r)\right| \leq C
$$

and

$$
\left|h_{\lambda} \frac{1-K_{\lambda}}{r^{2}}\right| \leq C
$$

for $r$ in $[0,1]$. Thus (35) gives:

$$
\left|-x^{2} K_{\lambda}^{\prime}-2 x\left(1-K_{\lambda}\right)\right| \leq C x^{4}
$$

Dividing this by $x^{3}$ yields

$$
\left|\frac{K_{\lambda}^{\prime}}{x}\right| \leq C x+2\left|\frac{1-K_{\lambda}}{x^{2}}\right|
$$

which by Proposition 10.1 gives the required estimate for $K_{\lambda}^{\prime}$.

\section{Estimates ON $[0, \infty)$}

In this section the estimates of Sections 7, 8, 9 and 10 are put together to show the convergence in $L^{2}$ as required for the proof of Theorem 5.5

Theorem 11.1. As $\lambda \rightarrow 0$ the following hold for the $L^{2}$ norms on $[0, \infty)$
1. $\left\|\frac{1}{r}\left(H_{\lambda}-H_{0}\right)\right\|_{2} \rightarrow 0$
2. $\left\|\frac{1}{r^{2}}\left(H_{\lambda}-H_{0}\right)\right\|_{2} \rightarrow 0$
3. $\left\|H_{\lambda}^{\prime \prime}-H_{0}^{\prime \prime}\right\|_{2} \rightarrow 0$ 
4. $\left\|H_{\lambda}^{\prime}-H_{0}^{\prime}\right\|_{2} \rightarrow 0$

5. $\left\|\frac{1}{r}\left(H_{\lambda}^{\prime}-H_{0}^{\prime}\right)\right\|_{2} \rightarrow 0$

In addition, for any $b>0$ the following holds:

$$
\int_{0}^{b}\left(H_{\lambda}-H_{0}\right)^{2} d r \rightarrow 0
$$

as $\lambda \rightarrow 0$.

Proof. For (1) let $\varepsilon>0$ and consider $C>0$ given by Proposition 10.1 and Corollary 9.3 such that:

$$
\left(H_{\lambda}-H_{0}\right)^{2} \leq C
$$

and for $\lambda$ small,

$$
\left|\frac{1}{r^{2}}\left(H_{\lambda}-H_{0}\right)\right| \leq C
$$

for $r$ in $[0,1]$. Choose $r_{0}$ such that $\max \left(\frac{\varepsilon}{C}, \frac{1}{\varepsilon}\right)<r_{0}$ and write:

$$
\begin{aligned}
\left\|\frac{1}{r}\left(H_{\lambda}-H_{0}\right)\right\|_{2}^{2} & =\int_{0}^{\varepsilon / C} \frac{1}{r^{2}}\left(H_{\lambda}-H_{0}\right)^{2} d r+\int_{\varepsilon / C}^{r_{0}} \frac{1}{r^{2}}\left(H_{\lambda}-H_{0}\right)^{2} d r+\int_{r_{0}}^{\infty} \frac{1}{r^{2}}\left(H_{\lambda}-H_{0}\right)^{2} d r \\
& \leq \varepsilon+\frac{C^{2}}{\varepsilon^{2}} \int_{\varepsilon / C}^{r_{0}}\left(H_{\lambda}-H_{0}\right)^{2} d r+C \int_{r_{0}}^{\infty} \frac{1}{r^{2}} d r \\
& \leq 2 \varepsilon+\int_{\varepsilon / C}^{r_{0}}\left(H_{\lambda}-H_{0}\right)^{2} d r .
\end{aligned}
$$

Now use Proposition 7.4.

The proof of (2) is similar.

For (3) write

$$
\begin{aligned}
\left\|H_{\lambda}^{\prime \prime}-H_{0}^{\prime \prime}\right\|_{L^{2}[0, \infty)} & =\left\|\frac{2 K_{\lambda}^{2} H_{\lambda}}{r^{2}}-\frac{2 K_{0}^{2} H_{0}}{r^{2}}-4 \lambda H_{\lambda}\left(1-\frac{H_{\lambda}^{2}}{r^{2}}\right)\right\|_{L^{2}[0, \infty)} \\
& \leq\left\|\frac{2 K_{\lambda}^{2} H_{\lambda}}{r^{2}}-\frac{2 K_{0}^{2} H_{0}}{r^{2}}\right\|_{L^{2}[0, \infty)}+4 \lambda\left\|H_{\lambda}\left(1-\frac{H_{\lambda}^{2}}{r^{2}}\right)\right\|_{L^{2}[0, \infty)} .
\end{aligned}
$$

For $\alpha$ and $r_{0}$ be as in Proposition 9.1. Then, using that $\left|H_{\lambda}(r)\right| \leq r$ for all $\lambda>0, r \geq 0$ and the estimate of Proposition 9.1,

$$
\begin{aligned}
4 \lambda\left\|H_{\lambda}\left(1-\frac{H_{\lambda}^{2}}{r^{2}}\right)\right\|_{L^{2}[0, \infty)} & \leq 4 \lambda\left\|H_{\lambda}\left(1-\frac{H_{\lambda}^{2}}{r^{2}}\right)\right\|_{L^{2}\left[0, r_{0}\right]}+8 \lambda\left\|H_{\lambda}\left(1-\frac{H_{\lambda}}{r}\right)\right\|_{L^{2}\left[r_{0}, \infty\right)} \\
& \leq 8 \lambda r_{0}+8 \lambda \alpha\left\|r e^{-\sqrt{\lambda} r}\right\|_{L^{2}\left[r_{0}, \infty\right)}
\end{aligned}
$$


which goes to zero as $\lambda \rightarrow 0$ by (22). Now for $\varepsilon>0$ let $\alpha$ and $r_{0}$ be as in Proposition 8.1. Let $r_{0}$ be the maximum of the $r_{0}$ given in Proposition 8.1 and the $r_{0}$ which satisfies

$$
\left\|\alpha \frac{e^{-r / 2}}{r}\right\|_{L^{2}\left[r_{0}, \infty\right)} \leq \varepsilon
$$

Since $K_{\lambda}$ converges to $K_{0}$ in $C\left[0, r_{0}\right]$ as $\lambda \rightarrow 0$, choose $\lambda_{0}$ to be the minimum of the $\lambda_{0}$ given in Proposition 10.1 and the $\lambda_{0}$ which satisfies:

$$
\left|K_{\lambda}(r)\right| \leq 2
$$

for all $r$ in $\left[0, r_{0}\right]$ and $\lambda$ in $\left[0, \lambda_{0}\right]$. Now for $B$ be as in Proposition $10.1, r_{1}=\min (1, B / \varepsilon)^{2}$ and for $\lambda \in\left[0, \lambda_{0}\right]$

$$
\begin{aligned}
\left\|\frac{2 K_{\lambda}^{2} H_{\lambda}}{r^{2}}-\frac{2 K_{0}^{2} H_{0}}{r^{2}}\right\|_{L^{2}[0, \infty)} \leq & \left\|\frac{K_{\lambda}^{2} H_{\lambda}}{r^{2}}\right\|_{L^{2}\left[0, r_{1}\right]}+\left\|\frac{K_{0}^{2} H_{0}}{r^{2}}\right\|_{L^{2}\left[0, r_{1}\right]} \\
& +\left\|\frac{K_{\lambda}^{2}\left(H_{\lambda}-H_{0}\right)}{r^{2}}\right\|_{L^{2}\left[r_{1}, r_{0}\right]}+\left\|\frac{\left(K_{\lambda}^{2}-K_{0}^{2}\right) H_{0}}{r^{2}}\right\|_{L^{2}\left[r_{1}, r_{0}\right]} \\
& +2\left\|\alpha \frac{e^{-r / 2}}{r}\right\|_{L_{2}\left[r_{0}, \infty\right)} \\
\leq & 2 B \frac{\varepsilon}{B}+2 B \frac{\varepsilon}{B} \\
& +\frac{4}{r_{1}^{2}}\left\|H_{\lambda}-H_{0}\right\|_{L^{2}\left[r_{1}, r_{0}\right]}+\frac{r_{0}}{r_{1}^{2}}\left\|K_{\lambda}^{2}-K_{0}^{2}\right\|_{L^{2}\left[r_{1}, r_{0}\right]} \\
& +2 \varepsilon .
\end{aligned}
$$

The last expression becomes smaller that $8 \varepsilon$ if $\lambda$ is small enough, by the uniform convergence of $K_{\lambda}$ to $K_{0}$ and $H_{\lambda}$ to $H_{0}$ on the interval $\left[r_{1}, r_{0}\right]$. Since $\varepsilon$ was arbitrary, this finishes the proof.

For (4), let $\varepsilon>0$ be arbitrary, fix $3 / 2<p<2$ and choose $r_{0}>0$ be the maximum of the $r_{0}$ in Proposition 9.5 and the $r_{0}$ which satisfies:

$$
\left\|\frac{\alpha}{r^{p-1}}\right\|_{L^{2}\left[r_{0}, \infty\right)}+\left\|\frac{\alpha}{r}\right\|_{L^{2}\left[r_{0}, \infty\right)}+\left\|1-H_{0}^{\prime}\right\|_{L^{2}\left[r_{0}, \infty\right)}<\varepsilon
$$


where $\alpha$ is the maximum of the constants which appear in Propositions 9.2 and 9.5. Let $\lambda_{0}$ and $\alpha$ be as in Proposition 9.5 and note that for $\lambda \in\left[0, \lambda_{0}\right]$ we have that:

$$
\left|\frac{H_{\lambda}^{\prime}}{r}-\frac{H_{\lambda}}{r^{2}}\right|=\left|\left(\frac{H_{\lambda}}{r}\right)^{\prime}\right| \leq \frac{\alpha}{r^{p}}
$$

thus

$$
\left|H_{\lambda}^{\prime}-\frac{H_{\lambda}}{r}\right| \leq \frac{\alpha}{r^{p-1}}
$$

Therefore

$$
\left|H_{\lambda}^{\prime}-H_{0}^{\prime}\right| \leq\left|H_{\lambda}^{\prime}-\frac{H_{\lambda}}{r}\right|+\left|\frac{H_{\lambda}}{r}-1\right|+\left|1-H_{0}^{\prime}\right| \leq \frac{\alpha}{r^{p-1}}+\frac{\alpha}{r}+\left|1-H_{0}^{\prime}\right| .
$$

Thus

$$
\left\|H_{\lambda}^{\prime}-H_{0}^{\prime}\right\|_{L^{2}\left[r_{0}, \infty\right)} \leq\left\|\frac{\alpha}{r^{p-1}}\right\|_{L^{2}\left[r_{0}, \infty\right)}+\left\|\frac{\alpha}{r}\right\|_{L^{2}\left[r_{0}, \infty\right)}+\left\|1-H_{0}^{\prime}\right\|_{L^{2}\left[r_{0}, \infty\right)}<\varepsilon
$$

Also,

$$
\left\|H_{\lambda}^{\prime}-H_{0}^{\prime}\right\|_{L^{2}\left[0, r_{0}\right]} \rightarrow 0 \text { as } \lambda \rightarrow 0
$$

by Poincare's Theorem (which applies by Part 3 and the estimate $H_{\lambda}^{\prime}(0)=H_{0}^{\prime}(0)=0$ for $\lambda$ small enough).

For (5) let $\varepsilon>0$ and choose $C$ by Proposition 10.3 to satisfy

$$
\frac{1}{r^{2}}\left(H_{\lambda}^{\prime}-H_{0}^{\prime}\right)^{2} \leq C
$$

for $r$ in $[0,1]$. Then

$$
\begin{aligned}
\left\|\frac{1}{r}\left(H_{\lambda}^{\prime}-H_{0}^{\prime}\right)\right\|_{2}^{2} & \leq \int_{0}^{\varepsilon / C} \frac{1}{r^{2}}\left(H_{\lambda}^{\prime}-H_{0}^{\prime}\right)^{2} d r+\int_{\varepsilon / C}^{\infty} \frac{1}{r^{2}}\left(H_{\lambda}^{\prime}-H_{0}^{\prime}\right)^{2} d r \\
& \leq \varepsilon+\frac{C^{2}}{\varepsilon^{2}} \int_{\varepsilon / C}^{\infty}\left(H_{\lambda}^{\prime}-H_{0}^{\prime}\right)^{2} d r .
\end{aligned}
$$

Now note that the last integral goes to zero by (4).

To show the last statement of the theorem use Proposition 10.3 at a small neighborhood of zero and Proposition 7.4 on the rest of the interval $[0, b]$.

Theorem 11.2. As $\lambda \rightarrow 0$ the following hold for the $L^{2}$ norms on $[0, \infty)$

1. $\left\|K_{\lambda}-K_{0}\right\|_{2} \rightarrow 0$,

2. $\left\|\frac{1}{r}\left(K_{\lambda}-K_{0}\right)\right\|_{2} \rightarrow 0$, 
3. $\left\|\frac{1}{r^{2}}\left(K_{\lambda}-K_{0}\right)\right\|_{2} \rightarrow 0$,

4. $\left\|K_{\lambda}^{\prime \prime}-K_{0}^{\prime \prime}\right\|_{2} \rightarrow 0$,

5. $\left\|K_{\lambda}^{\prime}-K_{0}^{\prime}\right\|_{2} \rightarrow 0$,

6. $\left\|\frac{1}{r}\left(K_{\lambda}^{\prime}-K_{0}^{\prime}\right)\right\|_{2} \rightarrow 0$,

Proof. For (1) write

$$
\left\|K_{\lambda}-K_{0}\right\|_{2}^{2}=\int_{0}^{r_{0}}\left(K_{\lambda}-K_{0}\right)^{2} d r+\int_{r_{0}}^{\infty}\left(K_{\lambda}-K_{0}\right)^{2} d r
$$

Now use Proposition 7.4 to see that the first integral goes to zero and Proposition 8.1 to choose $r_{0}$ large enough to make the second integral arbitrarily small.

For (2) write

$$
\left\|\frac{1}{r} K_{\lambda}-K_{0}\right\|_{2}^{2}=\int_{0}^{\varepsilon} \frac{1}{r^{2}}\left(K_{\lambda}-K_{0}\right)^{2} d r+\int_{\varepsilon}^{r_{0}} \frac{1}{r^{2}}\left(K_{\lambda}-K_{0}\right)^{2} d r+\int_{r_{0}}^{\infty} \frac{1}{r^{2}}\left(K_{\lambda}-K_{0}\right)^{2} d r .
$$

Now use Proposition 10.1 to choose $\varepsilon$ that makes the first integral arbitrarily small, Proposition 7.4 for the second integral and Proposition 8.1 for the third.

For (3) repeat the proof of (2).

For (4) equation (YMH-1) gives

$$
\begin{aligned}
\left\|K_{\lambda}^{\prime \prime}-K_{0}^{\prime \prime}\right\|_{2} & \leq\left\|\frac{H_{\lambda}^{2}-H_{0}^{2}}{r^{2}} K_{\lambda}\right\|_{2}+\left\|\left(H_{0}^{2}-1+K_{\lambda}^{2}+K_{\lambda} K_{0}+K_{0}^{2}\right) \frac{K_{\lambda}-K_{0}}{r^{2}}\right\|_{2} \\
& \leq C\left(\left\|\frac{H_{\lambda}^{2}-H_{0}^{2}}{r^{2}}\right\|_{2}+\left\|K_{\lambda}-K_{0}\right\|_{2}\right) \\
& \leq C\left(\left\|\frac{H_{\lambda}-H_{0}}{r^{3 / 2}}\right\|_{2}+\left\|K_{\lambda}-K_{0}\right\|_{2}\right)
\end{aligned}
$$

which goes to 0 by Proposition 11.1 and part (1) of this Theorem.

For (5) use Poincare's inequality and part (4) on a compact interval and the exponential decay on $K_{\lambda}^{\prime}-K_{0}^{\prime}$ of Proposition 8.2.

For (6) use Proposition 10.3 close to zero, Proposition 7.4 on a compact interval and the exponential decay at infinity, as above. 


\section{Arguments For ANy POSItive $\lambda$}

The arguments above have made a step from the $\lambda=0$ Yang-Mills-Higgs theory to $\lambda \neq 0$ Yang-Mills-Higgs theory. In particular it has been shown that the set

$$
\Lambda=\left\{\lambda \neq 0: Q_{\lambda}^{c_{\lambda}} \geq 0, \operatorname{Ker} Q_{\lambda}^{c_{\lambda}}=S_{\lambda}\right\}
$$

is nonempty.

Once away from zero, the norm

$$
\|(a, \phi)\|\left\|^{2}=\right\| \nabla_{A} a\left\|_{2}^{2}+\right\| \nabla_{A} \phi\left\|_{2}^{2}+\right\|[\Phi, a]\left\|_{2}^{2}+\right\| \phi \|_{2}^{2}
$$

becomes relevant on $T \mathcal{C}_{+}$and with respect to this norm $Q_{\lambda}^{c_{\lambda}}$ has property $(\dagger)$.

With this, and after repeating the arguments of sections 8, 9 and 10 with 0 replaced by $\sup \Lambda$, it can also show that the set $\Lambda$ is open. To show that $\Lambda$ is in fact closed will follow once the lowest non-zero eigenvalue of $Q_{\lambda}^{c_{\lambda}}$ is controlled by an increasing function of $\lambda$.

It is the intention of the authors to substantiate these claims in a paper currently in progress.

\section{REFERENCES}

[BL] Bourguignon J.-P. and H.B Lawson, Stability and Isolation Phenomena for Yang-Mills Fields, Comm. Math. Phys. 79, 189-230, 1981

[D1] Dostoglou, S. The Banach Manifold Structure on the Configuration Space of the Yang-Mills-Higgs Functional Preprint, available at http://www.math.missouri.edu/people/

[D2] Dostoglou S. Remarks on the Existence of Solutions of the Yang-Mills-Higgs Equations with Arbitrary Higgs Potential, Preprint available at http://www.math.missouri.edu/people/

[F] Floer, A. The configuration space of Yang-Mills-Higgs theory on asymptotically flat manifolds The Floer Memorial Volume, Progress in Mathematics 133, 43-75, Birkhäuser, 1995

[H] Higgs P. Spontaneous Symmetry Breakdown without Massless Boson Phys. Rev. 145 (1966), 11561163.

[JT] Jaffe, A. and C. H. Taubes, Vortices and Monopoles, Birkhauser, 1980.

[M] Maison D. Uniqueness of the Prasad-Sommerfield Monopole Solution, Nucl. Phys.B 182, 1981.

[P] Polyakov: JETP Letters 20, 1974.

[R] Rawnsley J. H. Existence and regularity of solutions of gauge field equations, Seminar Notes, School of Theoretical Physics, Dublin Institute for Advanced Studies, (1977). 
[RFS] Romanov V. N. and I. V. Frolov, A. S. Schwarz Spherically Symmetric Solitons Teor. Mat. Fiz. 37, 1978.

[ST] Sibner, L. and J. Talvachia The existence of non-minimal solutions of the Yang-Mills-Higgs equations over $\mathbf{R}^{3}$ with arbitrary positive coupling constant. Commun. Math. Phys. 162 (1994), 333-351.

[T1] Taubes, C.H. Stability in Yang-Mills Theories, Commun. Math. Phys. 91 (1983), 235 -263.

[T2] Taubes, C.H. Monopoles and Maps from $S^{2}$ to $S^{2}$; the Topology of the Configuration Space, Commun. Math. Phys. 95 (1984), 345-391.

[T3] Taubes C. H. Existence of a Non-Minimal Solutions to the SU(2) Yang-Mills-Higgs Equations, Part I Comm. Math. Phys. 86, 1982.

[T4] Taubes C. H. Min-Max Theory for the Yang-Mills-Higgs Equations Comm. Math. Phys. 97, 1985.

[tH] G. 't Hooft Magnetic monopoles in unified gauge theories Nucl. Phys. B 79, 1976, 276

[TFS] Tyupkin S. T. and V. A. Fateev, A. S. Schwarz Particle-like Solutions of the Equations of Gauge Theories Teor. Mat. Fiz. 26, 1976.

[WB] Wirzba, A. and H. Bang The mode spectrum and stability analysis of skyrmions on the 3-sphere Nuclear Phys. A 515 (1990), no 4, 571- 598.

Mathematical Sciences Building, University of Missouri-Columbia, Columbia MO 65211

E-mail address: giorgis@math.missouri.edu, stamatis@euler.math.missouri.edu 